\title{
Combining multiple structural inversions to constrain the solar modelling problem
}

\author{
G. Buldgen ${ }^{1}$, S. J. A. J. Salmon ${ }^{2}$, A. Noels ${ }^{2}$, R. Scuflaire ${ }^{2}$, J. Montalban ${ }^{3}$, V. A. Baturin ${ }^{4}$, P. Eggenberger ${ }^{5}$, \\ V. K. Gryaznov ${ }^{6,7}$, I. L. Iosilevskiy ${ }^{8,9}$, G. Meynet ${ }^{5}$, W. J. Chaplin ${ }^{1}$, A. Miglio ${ }^{1}$, A. V. Oreshina ${ }^{4}$, \\ O. Richard ${ }^{10}$, and A. N. Starostin ${ }^{11}$
}

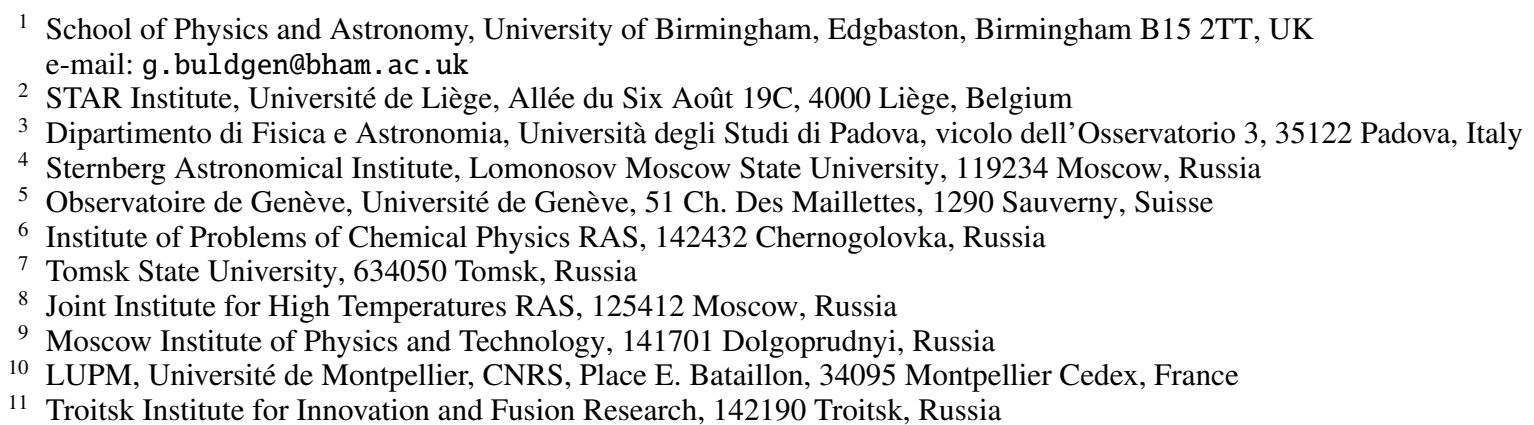

Received 27 July 2018 / Accepted 24 September 2018

\begin{abstract}
Context. The Sun is the most studied of all stars, which serves as a reference for all other observed stars in the Universe. Furthermore, it also serves the role of a privileged laboratory of fundamental physics and can help us better understand processes occuring in conditions irreproducible on Earth. However, our understanding of our star is currently lessened by the so-called solar modelling problem, resulting from comparisons of theoretical solar models to helioseismic constraints. These discrepancies can stem from various causes, such as the radiative opacities, the equation of state as well as the mixing of the chemical elements.

Aims. By analysing the potential of combining information from multiple seismic inversions, our aim is to help disentangle the origins of the solar modelling problem.

Methods. We combined inversions of the adiabatic sound speed, an entropy proxy and the Ledoux discriminant with other constraints such as the position of the base of the convective zone and the photospheric helium abundance. First, we tested various combinations of standard ingredients available for solar modelling such as abundance tables, equation of state, formalism for convection and diffusion and opacity tables. Second, we studied the diagnostic potential of the inversions on models including ad hoc modifications of the opacity profile and additional mixing below the convective envelope.

Results. We show that combining inversions provides stringent constraints on the required modifications to the solar ingredients, far beyond what can be achieved from sound speed inversions alone. We constrain the form and amplitude of the opacity increase required in solar models and show that a $15 \%$ increase at $\log T=6.35$ provides a significant improvement, but is insufficient on its own. A more global increase in the opacity, within the uncertainties of the current tables, coupled with a localized additional mixing at the bottom of the convective zone provides the best agreement for low-metallicity models. We show that high-metallicity models do not satisfy all the inversion results. We conclude that the solar modelling problem likely occurs from multiple small contributors, as other ingredients such as the equation of state or the formalism of convection can induce small but significant changes in the models and that using phase shift analyses combined with our approach is the next step for a better understanding of the inaccuracies of solar models just below the convective envelope.
\end{abstract}

Key words. Sun: helioseismology - Sun: fundamental parameters - Sun: oscillations - Sun: interior

\section{Introduction}

In the past decades, helioseismology has been one of the most successful fields of astrophysics. Its achievements stem from the excellent quality of the seismic data, allowing thorough comparisons between solar models and the Sun. These studies led to the precise determination of the position of the base of the solar convective zone (hereafter BCZ; Kosovichev \& Fedorova 1991; Christensen-Dalsgaard et al. 1991; Basu \& Antia 1997), the present day helium abundance in the convective zone (Vorontsov et al. 1991; Dziembowski et al. 1991; Basu \& Antia
1995; Richard et al. 1998; Vorontsov et al. 2013), unconstrained by photosphere spectroscopy, the determination of the radial profile of thermodynamical quantities inside the Sun (see e.g. Antia \& Basu 1994; Marchenkov et al. 2000, and references therein) and that of the solar rotation profile (Brown \& Morrow 1987; Kosovichev 1988; Schou et al. 1998; García et al. 2007).

These successes have paved the way for the asteroseismic analyses of solar-like oscillators and have indirectly established the study of stellar oscillations as the "golden path" to determine stellar fundamental parameters such as mass, radius and age. However, while the reliability of seismology as a test of 
solar and stellar structure is well established, the accuracy of stellar and solar models is still under question. In the solar case, the most tedious issue is linked to the revision of the abundance of heavy elements by Asplund et al. (2004, 2005, 2009), Caffau et al. (2011), Scott et al. (2015a,b) and Grevesse et al. (2015), which leads to a strong disagreement between solar models and helioseismology which, in turn leads to questions regarding the reliability of solar models. The main difficulty with this issue is that the observed discrepancies can originate from multiple sources. Amongst theses sources, the largest contributor is radiative opacity, which is thought to be underestimated for the physical conditions of the BCZ (Bailey et al. 2015; Iglesias 2015; Nahar \& Pradhan 2016; Pradhan \& Nahar 2018; Zhao et al. 2018; Pain et al. 2018), although the origin of the discrepancy is still under debate (Blancard et al. 2016; Iglesias \& Hansen 2017; Pain et al. 2017). However, other inputs of standard models can also contribute to the discrepancies, such as the equation of state, the formalism used for microscopic diffusion, the formalism of convection, or the nuclear reaction rates. Hence, the recipe of the standard model itself is a limiting factor to its expected accuracy. For example, the modelling of the BCZ, where the interplay between various non-standard processes, such as rotation, turbulence, and magnetism leads to the solar tachocline ${ }^{1}$. The current inability to accurately model these processes implies that the reliability of the standard solar models in these regions is questionable.

In previous papers (Buldgen et al. 2017a,c), we presented new structural inversions and showed how they could be used to reanalyse the solar modelling problem. We showed how combining all these inversions together can be used to offer stringent constraints on the processes and changes that can be applied to standard models to solve their current issue with seismic constraints. This is done by disentangling the competing effects of the various uncertainties on the physical processes inside the Sun. A similar analysis can be found in Ayukov \& Baturin (2011) and Ayukov \& Baturin (2017), in the latter study, the problem is redefined as an expanded solar calibration procedure.

We start in Sect. 2 by briefly presenting the ingredients of the current standard solar models with their respective uncertainties, and describe in Sect. 2.2 the set of models we computed using the Liège stellar evolution code (CLES) with various physical ingredients. We then present the adiabatic sound speed, entropy proxy and Ledoux discriminant inversions for our standard solar models and discuss the observed variations and other potential improvements that could lead to significant changes at the level of accuracy of helioseismic investigations. In Sect. 3, we compute solar models including ad hoc modifications, using parametric variations in the opacity profile and extra-mixing of the chemical elements below the convective zone to test the potential impact of such processes in the reconciliation of solar models with helioseismic constraints.

\section{Combined inversions for standard solar models}

In this section, we present structural inversion results of various thermodynamical quantities and discuss how their combination on a sample of standard solar models provides an in-depth analysis of the solar modelling problem.

\footnotetext{
1 The tachocline is defined, by analogy with the thermocline in oceanography, as the transition region in the Sun from the differentially latitudinal rotating convective envelope to the rigidly rotating radiative region (Spiegel \& Zahn 1992).
}

The use of structural inversions in global helioseismology is now a standard approach. In this work, we use the linear formulation of the inverse problem following the developments of Dziembowski et al. (1990), based on the variational analysis of the pulsation equations (see e.g. Lynden-Bell \& Ostriker 1967, and references therein). These developments lead to an inverse problem of the form

$$
\frac{\delta v_{n, \ell}}{v_{n, \ell}}=\int_{0}^{R} K_{s_{1}, s_{2}}^{n, \ell} \frac{\delta s_{1}}{s_{1}} \mathrm{~d} r+\int_{0}^{R} K_{s_{2}, s_{1}}^{n, \ell} \frac{\delta s_{2}}{s_{2}} \mathrm{~d} r+\mathcal{F}_{\text {Surf }},
$$

with $\frac{\delta v_{n, \ell}}{v_{n, \ell}}$ the relative frequency differences of degree $\ell$ and radial order $n, \frac{\delta s_{i}}{s_{j}}$ the relative differences in the acoustic variables for the considered formulation of the inverse problem, $K_{s_{i}, s_{j}}^{n, \ell}$ the kernel functions associated with the acoustic variables and $\mathcal{F}_{\text {Surf }}$ an operator describing the surface regions, inaccurately described with the hypotheses of the variational approach. We consider a description of these surface effects as a sixth-order polynomial as in Rabello-Soares et al. (1999). We use the SOLA (Substractive Optimally Localized Averages) inversion method from Pijpers \& Thompson (1994) to solve Eq. (1).

We use three pairs of acoustic variables for which the integral relations are solved. First, we carry out inversions of the squared adiabatic sound-speed, denoted $c^{2}$, using the $\left(c^{2}, \rho\right)$ structural kernels. Second, we carry out inversions for an entropy proxy (Buldgen et al. 2017c), denoted $S_{5 / 3}=\frac{P}{\rho^{5 / 3}}$, which stems from the Sackur-Tetrode equation for the entropy of a monoatomic non-degenerate ideal gas. The advantage of this quantity is that it reproduces the plateau-like behaviour expected from the entropy in fully mixed regions with a nearly adiabatic stratification such as the deep convective layers in the Sun. The height of this plateau is a key parameter of the solar convective zone. The height of the entropy proxy plateau is very sensitive to the temperature gradient in the radiative zone, which can thus be strongly constrained. For this inversion, we used the $\left(S_{5 / 3}, \Gamma_{1}\right)$ structural kernels. Finally, we also invert for the Ledoux discriminant, denoted $A=\frac{\mathrm{d} \ln P}{\mathrm{~d} \ln r}-\frac{1}{\Gamma_{1}} \frac{\mathrm{d} \ln \rho}{\mathrm{d} \ln r}$. The Ledoux discriminant has the advantage of being much more sensitive to local variations. It allows one to analyse the properties of both the temperature and chemical composition gradient at the $\mathrm{BCZ}$, offering strong constraints on potential extra-mixing processes.

Inversions were performed individually using the calibrated model as a reference to compute the structural kernels and individual frequencies. In Sect. 2.1, we describe how these quantities help us analyse the impact of various ingredients of standard solar models. The inversion results are presented for the whole set of models in Sect. 2.1, and we present in Table 1 other key helioseismic constraints such as the BCZ and the mass coordinate at this position as well as the helium and heavy elements abundances in the convective zone and the initial values of these quantities for each of our models.

The solar models were computed using the Liège stellar evolution code (CLES; Scuflaire et al. 2008b) and their eigenfrequencies were computed using the Liège oscillation code (LOSC; Scuflaire et al. 2008a). The seismic data used for the inversions is a combination of BiSON and MDI data (see Basu et al. 2009; Davies et al. 2014). Inversions were carried out using an adapted version of the InversionKit software (Reese et al. 2012). 
G. Buldgen et al.: Combining multiple structural inversions to constrain the solar modelling problem

Table 1. Parameters of the standard solar models used in this study.

\begin{tabular}{ccccccccccc}
\hline \hline$(r / R)_{\mathrm{BCZ}}$ & $(m / M)_{\mathrm{CZ}}$ & $Y_{\mathrm{CZ}}$ & $Z_{\mathrm{CZ}}$ & $Y_{0}$ & $Z_{0}$ & EOS & Opacity & Abundances & Diffusion & Convection \\
\hline 0.7224 & 0.9785 & 0.2363 & 0.01361 & 0.2664 & 0.01511 & FreeEOS & OPAL & AGSS09 & Thoul & MLT \\
0.7230 & 0.9786 & 0.2376 & 0.01355 & 0.2685 & 0.01523 & OPAL & OPAL & AGSS09 & Thoul & MLT \\
0.7272 & 0.9799 & 0.2368 & 0.01360 & 0.2682 & 0.01515 & CEFF & OPAL & AGSS09 & Thoul & MLT \\
0.7239 & 0.9790 & 0.2380 & 0.01355 & 0.2690 & 0.01524 & SAHA-S & OPAL & AGSS09 & Thoul & MLT \\
0.7215 & 0.9781 & 0.2350 & 0.01363 & 0.2647 & 0.01511 & FreeEOS & OP & AGSS09 & Thoul & MLT \\
0.7205 & 0.9777 & 0.2300 & 0.01372 & 0.2588 & 0.01520 & FreeEOS & OPLIB & AGSS09 & Thoul & MLT \\
0.7196 & 0.9779 & 0.2322 & 0.01368 & 0.2614 & 0.01516 & FreeEOS & OPAS & AGSS09 & Thoul & MLT \\
0.7224 & 0.9785 & 0.2363 & 0.01361 & 0.2664 & 0.01511 & FreeEOS & OPAL & AGSS09 & Thoul & FST \\
0.7235 & 0.9788 & 0.2373 & 0.01359 & 0.2648 & 0.01480 & FreeEOS & OPAL & AGSS09 & Paquette & MLT \\
0.7131 & 0.9757 & 0.2453 & 0.01809 & 0.2750 & 0.01999 & FreeEOS & OPAL & GN93 & Thoul & MLT \\
0.7157 & 0.9764 & 0.2465 & 0.01706 & 0.2765 & 0.01887 & FreEOS & OPAL & GS98 & Thoul & MLT \\
0.7248 & 0.9789 & 0.2338 & 0.01343 & 0.2639 & 0.01496 & FreeEOS & OPAL & AGSS09m & Thoul & MLT \\
0.7207 & 0.9780 & 0.2373 & 0.01393 & 0.2655 & 0.01547 & FreeEOS & OPAL & AGSS09Ne & Thoul & MLT \\
\hline
\end{tabular}

\subsection{Standard solar models and their physical ingredients}

The definition of the standard solar model stems from Bahcall et al. (1982) and defines a well-posed mathematical problem to compute a theoretical model of the Sun. A standard solar model is a $1 M_{\odot}$ stellar model, evolved up to the solar age, reproducing the current photospheric ratio of heavy elements over hydrogen, $Z / X$, the solar radius and the solar effective temperature (or luminosity). To fulfil these constraints, the models are built using three free parameters, the solar initial hydrogen and heavy elements abundance values and the mixing length parameter of convection. For the calibrations considered in this paper, we used the solar parameters of Mamajek et al. (2015).

In addition to the mathematical set-up of the problem, standard models are also defined by a set of physical ingredients, such as the metal mixture composition of the stellar plasma, the equation of state, the radiative opacities, and the nuclear reaction rates. The only transport processes included in standard solar models are thermal convection and microscopic diffusion, often using rather simple approaches. Convection follows local and simplified formalisms, such as the standard mixing length theory (MLT; Cox \& Giuli 1968) or the Full Spectrum of Turbulence model (FST) of Canuto \& Mazzitelli (1991), Canuto \& Mazzitelli (1992) and Canuto et al. (1996), while diffusion often uses approximations such as in Proffitt \& Michaud (1991) and Thoul et al. (1994).

This representation gives a satisfactory agreement with the Sun; however ,it is still uncertain. The modelling of the interaction between various physical processes such as rotation, magnetism, and turbulence at the $\mathrm{BCZ}$, the radiative opacities and our depiction of convection in the upper layers of the solar envelope can be listed as the most uncertain aspects of the present state of solar modelling. However, other key ingredients of the internal solar structure such as the equation of state and the hypotheses we use to compute the diffusion of chemical elements and the cross sections of some nuclear reactions which still present large uncertainties may also have a significant impact at the level of precision of helioseismic constraints (see Boothroyd \& Sackmann 2003, and references therein for a discussion on some specific ingredients). In addition, improving the outer layers of the solar models can also have a slight effect on the solar modelling problem, as discussed in Gabriel (1996) and Schlattl et al. (1997), and the importance of the inclusion of turbulent pressure in the modelling of the outer convective layers and the traces it could leave in seismic inversions could be investigated (see e.g. Houdek et al. 2017; Sonoi et al. 2017).

Moreover, the solar standard model neglects effects of physical processes such as rotation, the magnetic field, mass loss, internal gravity waves, compressible turbulent convection, or overshooting $^{2}$ and is unidimensional while some of the mentioned processes are intrinsically not. It is unable to reproduce the lithium depletion and the departures from spherical symmetry observed in the Sun. Some refinements to the transport of chemicals by diffusion are also often missing, such as the effects of radiative levitation or partial ionization. These effects have been studied by Turcotte et al. (1998) and Gorshkov \& Baturin (2010), amongst others. Other effects, such as the fineness of the opacity tables (see e.g. Le Pennec et al. 2015) or the choice of low-temperature opacity tables (Guzik \& Mussack 2010) will also impact the structure of solar models at a level seen by helioseismology. The impact of these non-standard processes is expected to be small, but likely not negligible.

\subsection{Set of models and inversion results}

To compare our standard models, we chose to keep one set of ingredients as reference and plot all the other inverted profile in figures including this specific reference in order to see directly the effects of various ingredients. We used a model built using the AGSS09 abundances (Asplund et al. 2009), the FreeEOS equation of state (Irwin 2012), the opal opacities (Iglesias \& Rogers 1996), the mixing-length theory of convection (Cox \& Giuli 1968), the formalism for microscopic diffusion by Thoul et al. (1994), and the nuclear reaction rates from Adelberger et al. (2011). All models also include effects of conduction from Potekhin et al. (1999) and from Cassisi et al. (2007), as well as low-temperature opacities from Ferguson et al. (2005). We used grey atmosphere models in the Eddington approximation in all our models.

We subdivided our comparisons into four main effects: changing the equation of state, changing the opacity tables, changing the abundances, and changing the formalism for convection and diffusion. Each of these effects is respectively represented as a subpanel in Figs. 1, 2 and 3 illustrating the inversions of the squared adiabatic sound speed, the entropy proxy and the

\footnotetext{
2 Here we denote as overshooting the extent of the convective region beyond the formal Schwarzschild limit derived from the local convection theory used in the model.
} 

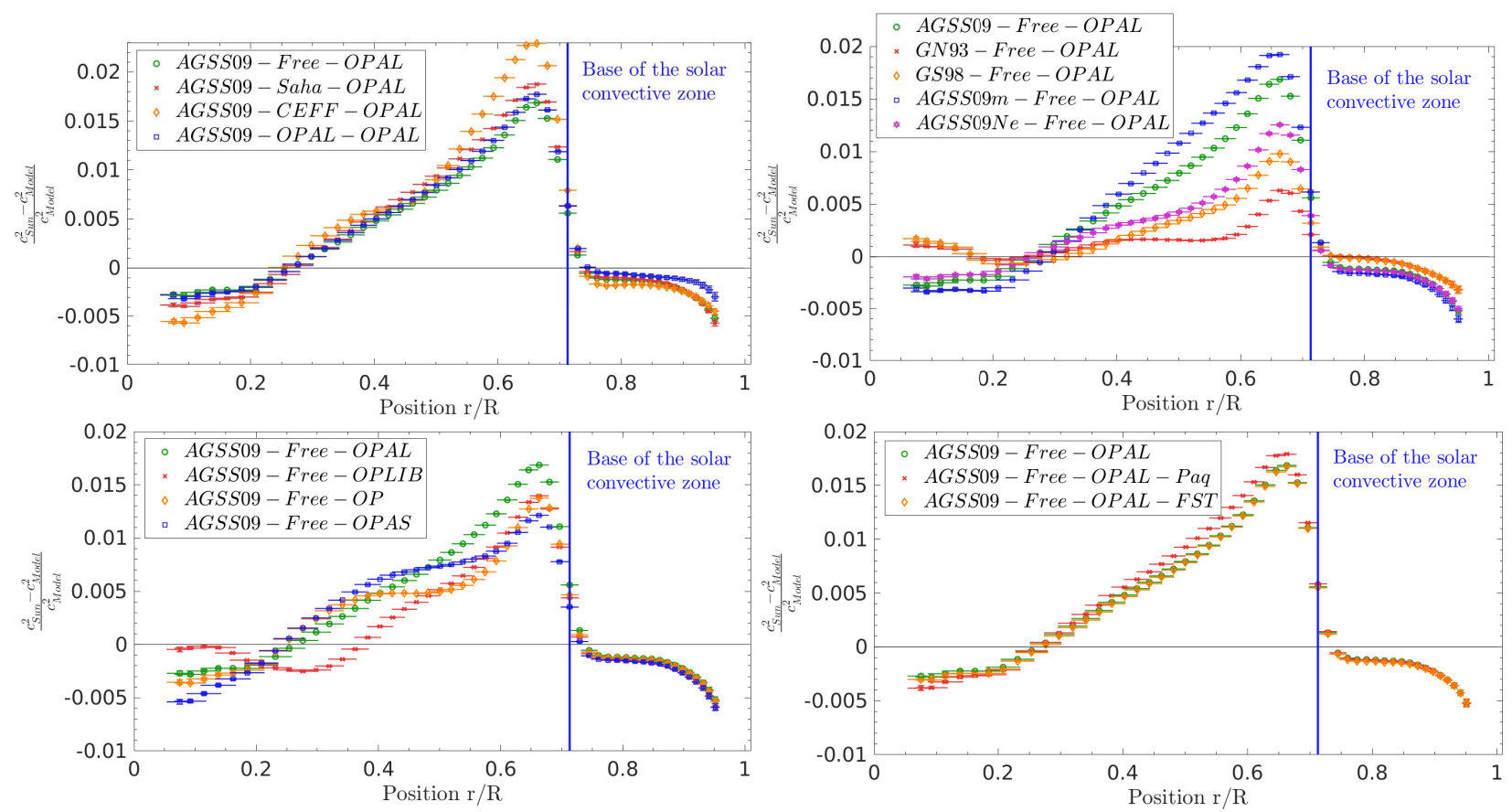

Fig. 1. Relative differences in squared adiabatic sound speed between the Sun and calibrated solar models. Upper left: effects of the equation of state. Upper right: effects of abundance variations. Lower left: effects of the opacity tables. Lower right: effects of changing the diffusion coefficients and the treatment of convection.
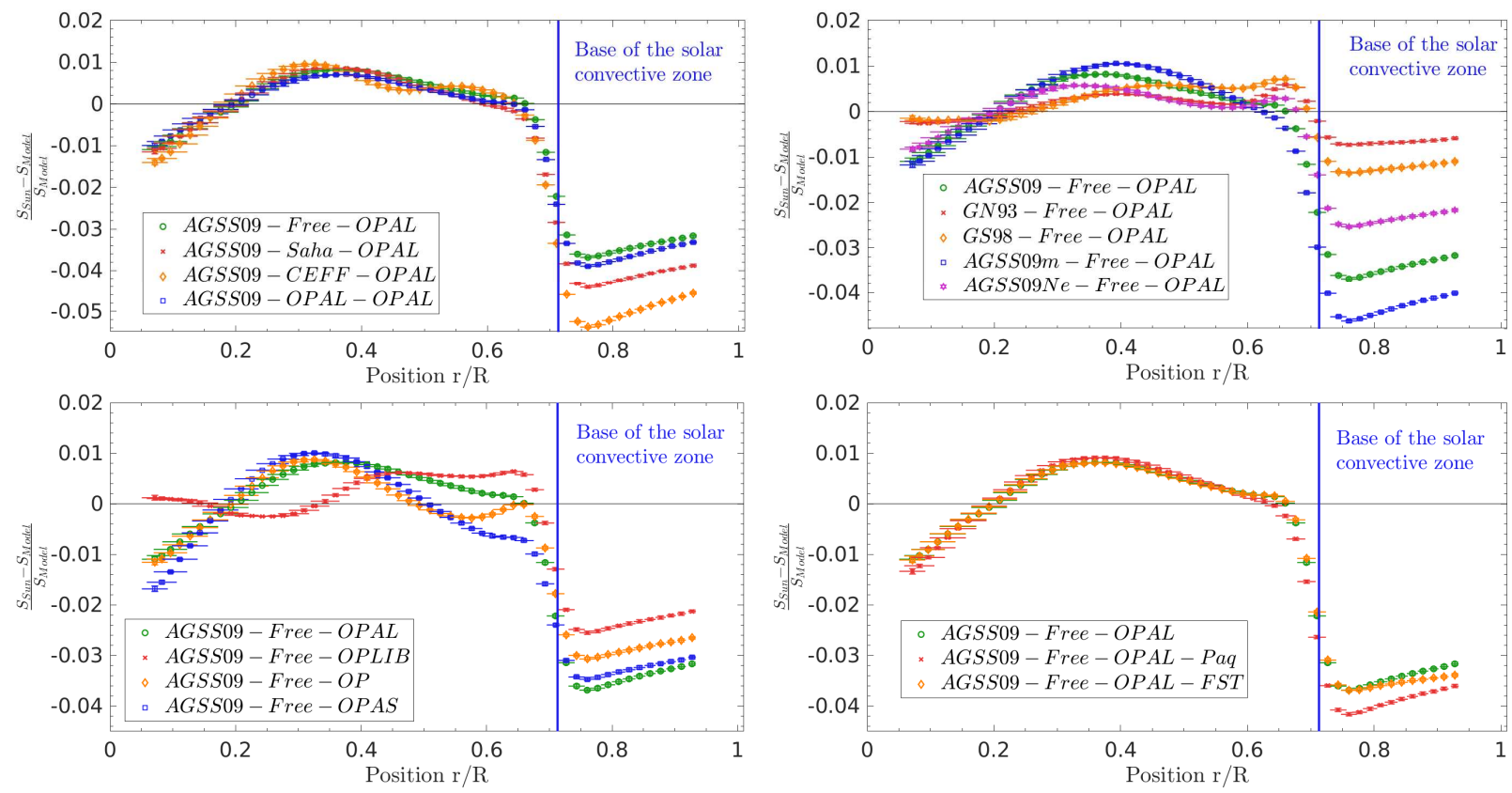

Fig. 2. Relative differences in entropy proxy, $S_{5 / 3}$, between the Sun and calibrated solar models. Upper left: effects of the equation of state. Upper right: effects of abundance variations. Lower left: effects of the opacity tables. Lower right: effects of changing the diffusion coefficients and the treatment of convection.

Ledoux convective parameters for each model of our sample. We use the SAHA-S (Gryaznov et al. 2006, 2013; Baturin et al. 2013), the OPAL (Rogers \& Nayfonov 2002), the FreeEOS and the CEFF equations of state (Christensen-Dalsgaard \& Däppen 1992; Irwin 2012) to analyse the variations induced by changing the equation of state in the solar models. The EOS-tables used in the model computations are initially defined with the hydrogen $X$ and the total heavy elements mass fraction $Z$ as parameters of the chemical composition. To test standard opacity tables, we used models built with the OPAL, OPAS (Mondet et al. 2015), OPLIB (Colgan et al. 2016), and OP (Badnell et al. 2005) opacity tables. For the abundances, we used models built with the former GN93 and GS98 abundances (Grevesse \& Noels 1993; Grevesse \& Sauval 1998) and models built with the more recent AGSS09 abundances. We also computed one table for which the abundances of $\mathrm{C}, \mathrm{N}, \mathrm{O}, \mathrm{Ne}$ and $\mathrm{Ar}$ were changed to the meteoritic values, as was done in Serenelli et al. (2009), denoted AGSS09m and one for which the recently suggested $40 \%$ neon 

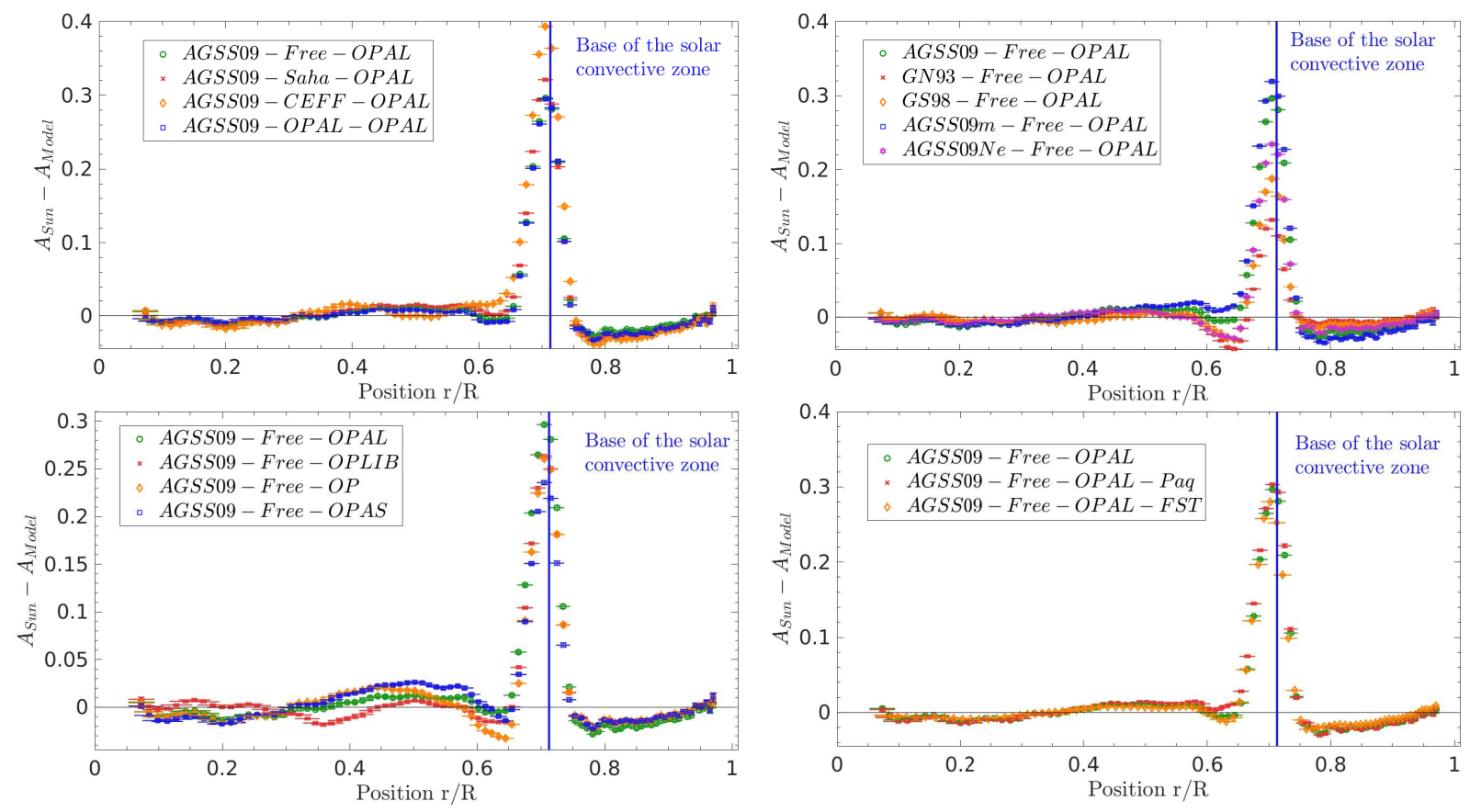

Fig. 3. Relative differences in Ledoux discriminant, $A$, between the Sun and calibrated solar models. Upper left: effects of the equation of state. Upper right: effects of abundance variations. Lower left: effects of the opacity tables. Lower right: effects of changing the diffusion coefficients and the treatment of convection.

abundance increase was taken into account (Landi \& Testa 2015; Young 2018), denoted AGSS09Ne. For each of these composition tables, the solar $Z / X$ ratio to be reproduced was adapted accordingly and opacity tables were recomputed for each abundance table. Finally, we also considered using the diffusion coefficients from Paquette et al. (1986) instead of those from Thoul et al. (1994) and the FST formulation of Canuto et al. (1996) in addition models computed using the classical mixinglength theory (Cox \& Giuli 1968).

The physical ingredients of the models are summarized in Table 1 alongside the photospheric helium and heavy-elements abundances, the position of their BCZ, the mass coordinate at this position and their initial heavy-elements and helium abundances. The position of the BCZ can be directly compared to the helioseismic value of 0.713 (Basu \& Antia 1997). As for the photospheric helium abundance, we consider that a value above 0.245 is acceptable as it agrees with intervals found in most recent studies (Vorontsov et al. 2013, 2014) and the usual value of 0.2485 (Antia \& Basu 1994; Basu \& Antia 2004). The parameters of the reference AGSS09-FreeEOS-OPAL model mentioned earlier are given in the first line of Table 1 and the inversion results of this model are plotted in green throughout the paper and are referred to in the plots as AGSS09-Free-Opal.

The inversions have been computed using the SOLA method (Pijpers \& Thompson 1994) and the guidelines of Rabello-Soares et al. (1999) using the software and equations of Buldgen et al. $(2017 \mathrm{a}, \mathrm{c})$. The results are shown in Fig. 1 for the squared adiabatic sound speed, in Fig. 2 for the entropy proxy, $S_{5 / 3}$; and in Fig. 3 for the Ledoux discriminant, $A$.

\subsection{Discussion}

From a first glance at the inversion results presented in Figs. 1, 2 and 3 , the first unsurprising conclusion that can be drawn is that to date there is no combination of opacity tables and equation of state that can reconcile standard models computed using the AGSS09 abundances with helioseismic results. Moreover, it should be noted that using more recent opacity tables like the OP, OPAS, or OPLIB tables does not lead to an unequivocal improvement of the solar modelling problem.

\subsubsection{Changing the opacity tables}

We first study the effect of the opacity tables. The lower left panel of Figs. 1, 2 and 3 show that the inversion results are usually better when using more recent opacity tables, especially for sound speed. However, as seen from the $Y_{S}$ values in Table 1, this improvement is mitigated by the large decrease in the helium abundance in the convective zone. This effect is particularly strong for the OPLIB tables which give opacity values significantly that are lower than all the other tables in the radiative zone. This implies an increase in the initial hydrogen abundance in the calibrated model to compensate for the opacity decrease, which in turn leads to a decrease in the helium abundance. This effect is also seen in the OP and OPAS models, which all have a higher hydrogen abundance than the OPAL value.

Some of these differences can be attributed to the various equations of state used in the computation of these opacity tables. It will control the ionization stage of the elements and hence the radiative opacity. To obtain the ionization stage and ions distribution, a basic thermodynamic model is needed. It is known that the OPAL opacities are based on the "physical picture" approach (Rogers \& Nayfonov 2002) while the OP opacity tables used the so-called "chemical picture", and are essentially based on the MHD EOS code (Hummer \& Mihalas 1988; Mihalas et al. 1988, 1990; Däppen et al. 1988). The OPAS and OPLIB opacities used their own equations of state, also based on the chemical picture. It is interesting to note that the OPAS and OPLIB tables both find a slightly lower opacity 
in the lower radiative region, which disagrees with previous tables.

In addition, differences can also be observed in the number of metals considered. For example the OPAL opacities consider 21 elements, the OP tables consider 17 elements while the OPLIB and OPAS consider 30 and 22 elements respectively.

\subsubsection{Changing the equation of state}

By testing various equations of state, it can be seen that the inversion results are significantly improved when using the OPAL, FreeEOS or SAHA-S equation of state when compared to the CEFF equation of state (see the upper-left panel of Figs. 1, 2 and 3 ). However, differences in the chemical composition of the convective envelope can be seen between the models. In that sense, the SAHA-S and OPAL models seem to be very similar, with the only exception being the position and mass coordinate of the convective envelope. The FreeEOS and CEFF models, on the contrary, show larger discrepancies. In the case of FreeEOS, the differences are essentially found in the present helium abundance in the convective zone, while the BCZ is very similar. For CEFF, the differences are more striking and are seen in every parameter of the envelope and inverted profiles.

Intrinsic differences in EOS quantities may be related to the different "first-principle" approaches, and to the additional terms and refinements which enter the thermodynamic potential and the numerical techniques used to compute the equation of state. The OPAL equation of state and the OPAL opacity tables are based on the physical picture. In this case, only fundamental constituents are used to compute the effects of inter-particle interactions ab initio. The grand Gibbs potential as a function of activities is used to calculate the specific thermodynamic quantities. All other current equations of state considered here (CEFF, FreeEOS, SAHA-S) are based on the free-energy-minimization approach in the chemical picture. The chemical picture provides the detailed ion distributions needed for spectroscopy and opacity calculations, as well as a thermodynamically consistent EOS, for a mixture of many "almost-ideal" reacting components. Within the same physical assumptions, both approaches should provide the same thermodynamics description. However, some differences are always observed in practice.

In the weakly non-ideal approximation, a contribution of Coulomb inter-particle interactions can be added to the thermodynamic potential as a separate term obtained from the results of an external calculation. In the early EOS formalisms, such as FreeEOS, CEFF, and MHD EOS, the Coulomb correction was added in the form of a simplified (linear) Debye-Hückel model or some generalization of it. In more advanced approaches, the Debye-Hückel contribution is obtained as a result of the perturbation procedure in the virial expansion and sequential generation of an activity expansion (Rogers \& DeWitt 1973) as used in the physical picture of the OPAL EOS. The same ringapproximation of the Debye-Hückel term has been used in the chemical picture of the SAHA-S EOS (Gryaznov et al. 2004).

Several additional thermodynamic corrections are needed for precise astrophysical modelling, such as radiative pressure, electron degeneracy, relativistic corrections, etc. They are treated as additional terms or extra corrections in the physical and the chemical approaches. The SAHA-S equations of state are the most recent chemical picture equations of state and they incorporate all principal results from quantum-statistical mechanics including Coulomb corrections and partition functions. This leads to the expectation that differences between the SAHA-S and OPAL equations of state should be minimal in our studies.

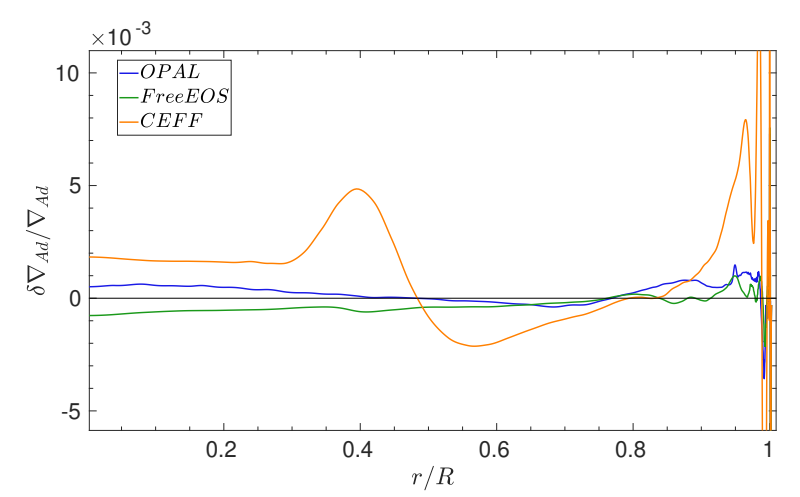

Fig. 4. Relative differences in adiabatic gradient between the CEFF, OPAL, and FreeEOS equation of state with respect to the SAHA-S equation of state, taken here as reference. $\delta \nabla_{\mathrm{Ad}} / \nabla_{\mathrm{Ad}}=\left(\nabla_{\mathrm{Ad}}^{\mathrm{EOS}}-\right.$ $\left.\nabla_{\mathrm{Ad}}^{\mathrm{SAHA}-\mathrm{S}}\right) / \nabla_{\mathrm{Ad}}^{\mathrm{SAHA}-\mathrm{S}}$, with EOS denoting either CEFF, FreeEOS, or the OPAL equation of state.

To illustrate the differences between the various equations of state, we plot in Fig. 4 an example of differences in thermodynamic quantities, namely the relative differences of the adiabatic gradient obtained with each equation of state from the input profiles of the density, temperature, and hydrogen and heavy element abundances, assuming an AGSS09 chemical mixture. Again, the CEFF equation of state is showing divergences when compared to all other equations. The reasons for these differences have not been carefully studied yet, but these discrepancies can already be seen at the beginning of the main sequence, and they certainly influence the evolution of the BCZ and thus the settling rates of chemical elements in the models.

As an additional test, we computed models with similar initial parameters with each equation of state to gain insight into what could be causing the observed differences. This analysis further showed that the OPAL and SAHA-S models were very similar, as both evolutionary sequences lead to similar parameters of the convective zones for both models. We could also observe that the FreeEOS model always showed a higher position of the envelope throughout the evolution and thus leads to a more effective diffusion. This lowers the hydrogen abundance in most of the stellar layers, which implies a lower opacity and thus a higher luminosity at the solar age. To recover the solar luminosity in a calibration, a higher metallicity is required. Since $Z / X$ is imposed for the calibration, $X$ must also be slightly increased and the resulting helium abundance is lower. As for the CEFF model, the behaviour of the settling rate is more complicated as the properties of the convective envelope are very different, probably as a consequence of the discrepancies in thermodynamic quantities which in turn influence the mean molecular weight gradient in the radiative zone and hence the luminosity. This last deduction remains speculative, however, and further dedicated studies are required to pinpoint the origin of these differences and what they imply for the solar modelling problem.

\subsubsection{Changing diffusion and convection}

Small variations can also be seen when using the Paquette et al. (1986) coefficients for diffusion. It appears that they lead to a slightly larger disagreement with the Sun, although not as significant as that using the CEFF equation of state. The effect is a direct consequence of the fact that the Paquette et al. (1986) coefficients lead to a less efficient transport of the chemical elements, which induces a slightly lower contrast in chemical 
composition between the radiative and convective zones which also leads to a less steep temperature gradient. Both these effects imply a slightly higher entropy plateau and small changes in $c^{2}$ and $A$ just below the convective envelope.

The physical origin of the differences between the Thoul et al. (1994) and Paquette et al. (1986) formalism, as presented in this study, is that for the latter case, the diffusion coefficients are computed from the collision integrals using screened Coulomb potentials, whereas the approach of Thoul et al. (1994) used a cut-off length for the Coulomb interaction placed at the Debye length. In their paper, Paquette et al. (1986) also argue that, in addition to being more realistic, screened Coulomb potentials are used to mimic to some extent multiparticle collisions.

Similarly, the model computed using the FST formulation of convection, denoted AD1-Free-OPAL-FST in Figs. 1, 2, and 3 , shows very similar results to the MLT model. The only difference is found in the behaviour of the entropy proxy plateau, which is flatter than with the MLT formulation and hence in better agreement with physical expections. This seems to imply that the FST formulation, which considers a whole spectrum of sizes for the convective elements, shows a better agreement with the entropy gradient in the surface region of the Sun and that the resulting surface effect could be smaller. Similar behaviour has been observed with MLT models complemented in the layers close to the surface by a $T(\tau)$ law from Vernazza et al. (1981). Further tests with models patched to averaged hydrodynamical simulations and various boundary conditions are required to check this behaviour to see if other contributors to the surface effect, such as the non-adiabaticity of the pulsation frequencies, could also induce similar trends.

\subsubsection{Changing the metal mixture}

The uppper right panel of Figs. 1, 2 and 3 illustrate the effects of abundance changes in standard solar models. The GN93 and GS98 models illustrate the agreement obtained with previous photospheric abundance tables derived from 1D empirical atmospheric models. From the adiabatic sound speed inversions, the depiction of the problem is quite straightforward; the large discrepancies resulting from the decrease in heavy elements abundances which strongly reduces the opacity in the radiative zone. From the entropy proxy and Ledoux discriminant inversions, other features start to appear, such as the small deviation of these models around 0.65 solar radius that is not present in models computed with the recent abundances. As noted in Buldgen et al. (2017a), this small deviation is the signature of a slightly too steep temperature gradient at this depth and perhaps a hint that the metallicity is indeed too high in these models ${ }^{3}$. In addition to the GN93 and GS98 abundance tables, we also plot the inversion results for models computed using the meteoritic abundances for all elements except for the volatile elements $\mathrm{C}, \mathrm{N}, \mathrm{O}, \mathrm{Ne}$, and $\mathrm{Ar}$, as in Serenelli et al. (2009), denoted AGSS09m. Using this modified abundance table, we find a slightly larger disagreement than with the standard AGSS09 table, similarly to their paper. Finally, the modified abundance table, denoted here AGSS09Ne, which includes the $40 \%$ increase in neon over oxygen determined independently by Landi \& Testa (2015) and Young (2018) seems to perform much better. This is not surprising since neon is the third contributor to the opacity after oxygen and iron at the BCZ (e.g.

\footnotetext{
3 However, it should be noted that efficient chemical mixing can lead to a less steep temperature gradient in these regions and that this feature could be erased with an ad hoc modification of the models.
}

Basu \& Antia 2008; Blancard et al. 2012). However, it seems that including this neon increase generates a similar behaviour to that of the GS98 models, which indicates that the steepening of the temperature gradients induced by the neon increase must somehow be mitigated. The idea of compensating for the decrease in oxygen through an increase in neon had already been presented in multiples studies (see e.g., Antia \& Basu 2005; Basu \& Antia 2008; Zaatri et al. 2007), although the increase was pushed to ten times the value found in recent studies and was adjusted in order to recover the agreemeent between AGSS09 models and helioseismic constraints. We come back to this in Sect. 3.2. Moreover, the heavy elements abundance tables have their own uncertainties which lead to an overall uncertainty of around $10 \%$ on the solar metallicity value (N. Grevesse, priv. comm.). This could significantly affect the solar modelling problem and emphasizes the need to constantly improve the precision of these measurements, as they are a key ingredient for solar and stellar modelling.

\section{Modified solar models}

In addition to standard solar models, we also analysed the changes in the various inverted profiles that could be obtained when applying ad hoc modifications to some key ingredients of the models. From Nahar \& Pradhan (2016) and Pradhan \& Nahar (2018), an increase in the mean Rosseland opacity cannot be excluded from future calculations. Additional experimental results from the Lawrence Livermore National Laboratory are in preparation and could independently confirm the results of the Sandia Z-pinch measurements (Bailey et al. 2015) of the spectral opacity of iron. This increase in the mean Rosseland opacity was recently estimated to be $9 \%$ at a temperature of $\log T \approx 6.3$ in preliminary calculations but higher values could be expected from more accurate computations (Zhao et al. 2018). The exact origin of these opacity underestimations may stem, at least partially, from inaccurate atomic data for certain key contributors such as iron, silicium, sulfur and to a lesser extent magnesium, oxygen and neon. In addition to the opacity problem, we found in our previous study that the discrepancies in the Ledoux discriminant observed in the tachocline could be reduced by adding a diffusive extra mixing supposed to mimic the effects of turbulence in a very thin region below the formal Schwarzschild boundary (Gabriel 1997; Brun et al. 1999, 2002). In addition to the chemical mixing, the behaviour of the temperature gradient, as it changes from the adiabatic temperature gradient to the radiative gradient, is also a source of uncertainty. The modelling of the overshooting region at the BCZ can be treated in various ways, none fully satisfactory, using a diffusive or instantaneous mixing that considers the temperature gradient to be adiabatic or radiative. In the next sections, we test the impact of opacity modifications and the impact of extramixing below the formal Schwarzschild boundary on the inversion results of $c^{2}, S_{5 / 3}$ and $A$. We will comment further on the overshooting problem in Sect. 3.2 and discuss how additional seismic constraints can be used to further constrain the temperature gradient transition and the type of mixing at the $\mathrm{BCZ}$.

\subsection{The opacity problem}

The main issue with testing changes in the opacity profile is that the degrees of freedom of the solar problem increase tremendously, as any type of change which can have various impacts on the models can be considered. We limited ourselves to changes motivated by physical considerations and discussions with 

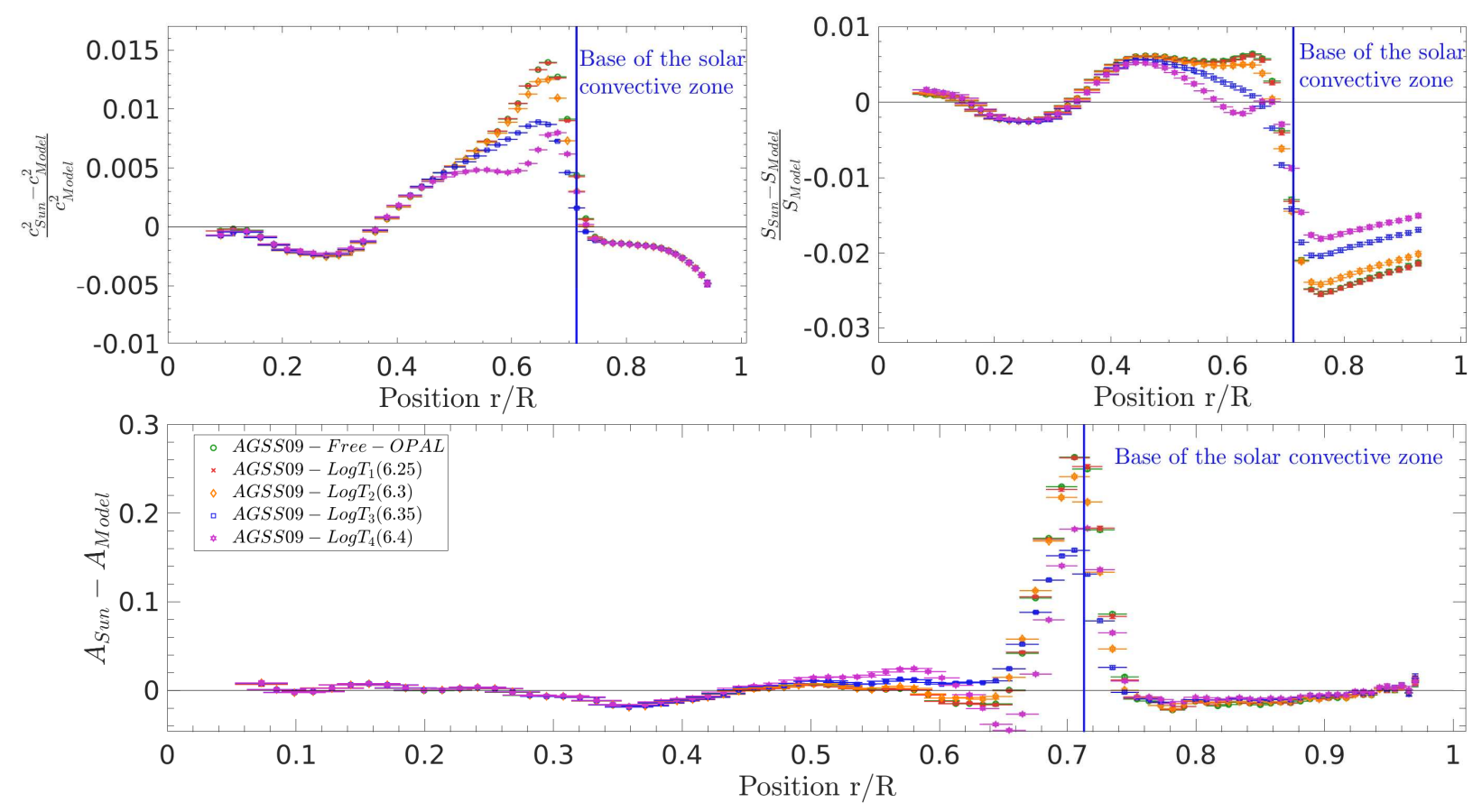

Fig. 5. Inversion results for models with modified opacities including a Gaussian peak of fixed height and width at various temperatures (see text for details). Upper left: relative differences in squared adiabatic sound speed. Upper right: relative differences in entropy proxy, $S_{5 / 3}$. Lower panel: differences in Ledoux discriminant, $A$.

Prof. Anil Pradhan. First, we started by investigating the impact of a Gaussian increase at various temperatures near the $\mathrm{BCZ}$, using the AGSS09 abundance tables, the FreeEOS equation of state and the OPLIB opacity tables as reference ingredients. Each model was recalibrated using a modified mean Rosseland opacity to grasp the effects over the evolution. The modification to the opacity profile is applied as a relative increase factor to the mean Rosseland opacity as

$\kappa^{\prime}=\left(1+f_{\kappa}\right) \kappa$,

with $\kappa$ the original mean Rosseland opacity, $f_{\kappa}$ the function defining the opacity modification and $\kappa^{\prime}$ the resulting modified opacity which is applied similarly during the whole evolution. At first, we start with a very narrow Gaussian increase for $f_{k}$ with

$f_{\kappa}=A \exp -\frac{\left(\log T-\log T_{\mathrm{Ref}}\right)^{2}}{2 \Delta^{2}}$,

with $A$ the amplitude of the increase, $\log T_{\text {Ref }}$ the peak temperature of the Gaussian and $\Delta$ its standard deviation. More complex modifications will also be considered (see Fig. 8) and applied similarly to the original mean Rosseland opacity profile.

We tested four temperatures for the peak of the Gaussian increase, namely $\log T_{\text {ref }}=6.25,6.30,6.35$, and 6.4 (denoted as $T_{1}, T_{2}, T_{3}$ and $T_{4}$, respectively) with maximum amplitude coefficients of $10 \%$, decreasing to $3 \%$ at 0.05 dex from the centre of the Gaussian. The results are shown in Fig. 5 and prove that the location of the peak has a large impact on the inverted profiles. At $\log T_{1}=6.25$ (red), the peak has almost no effect. Closer to the $\mathrm{BCZ}$, at $\log T_{2}=6.3$ (orange), the models start to show a slightly better agreement for all quantities. At $\log T_{3}=6.35$ (blue), the changes are drastic, with a large decrease in the height of the entropy plateau, changes in adiabatic sound speed and in the convective parameter at the BCZ. When the peak is moved to $\log T_{4}=6.4$ (purple), a slight improvement is observed for the entropy plateau and for the sound speed profile, but additional deviations are seen in the profile of the Ledoux discriminant around 0.65 solar radii. From Buldgen et al. (2017a), we could determine that this results from a too steep temperature gradient, which is not seen in the $S_{5 / 3}$ and $c^{2}$ inversions. It is also worth noting the similarities between the profiles of this model and that of a GN93 model built with the OPAL opacities, illustrating the degeneracy of the solar modelling problem in terms of abundances and opacities. We conclude that the optimal positioning for the opacity peak is at $\log T=6.35$, corresponding to the temperature of an iron opacity peak, which could be the source of the discrepancies.

In addition to the position in temperature, we tested the importance of the width of the Gaussian increase in opacity. We considered a position for the peak at $\log T=6.35$ and a maximum increase of $13 \%$ for all models shown in Fig. 6 . We started with $\Delta_{1} \approx 0.03$ (red), which allows for a decrease to $3 \%$ at 0.05 dex from the peak, then slightly modified the decrease with $\Delta_{2} \approx 0.032$ (orange) and $\Delta_{3} \approx 0.036$ (blue). These values imply that at 0.05 dex from the peak temperature, the opacity increased by $3.6 \%$ and $4.4 \%$, respectively. These variations are intentionally quite small and as expected, do not have a large impact on the models with the exception of $\Delta_{3}$, which starts showing slight differences that can arguably not be imputed to numerical uncertainties. It is however interesting to note that none of the models has led to an improvement in the convective zone helium abundance with respect to the threshold value of 0.245 we chose, while some can significantly reduce the discrepancies in the position of the $\mathrm{BCZ}$, the height of the entropy plateau and the sound speed profile compared to the standard AGSS09 model (see the first line of Table 1).

Similarly, we tested the impact of the maximum height of the Gaussian increase in opacity. We considered a position at $\log T=6.3$ and analysed the importance of increasing the height of the peak while simultaneously reducing its width to ensure that its amplitude was reduced to $3 \%$ at 0.05 dex from the peak 
G. Buldgen et al.: Combining multiple structural inversions to constrain the solar modelling problem
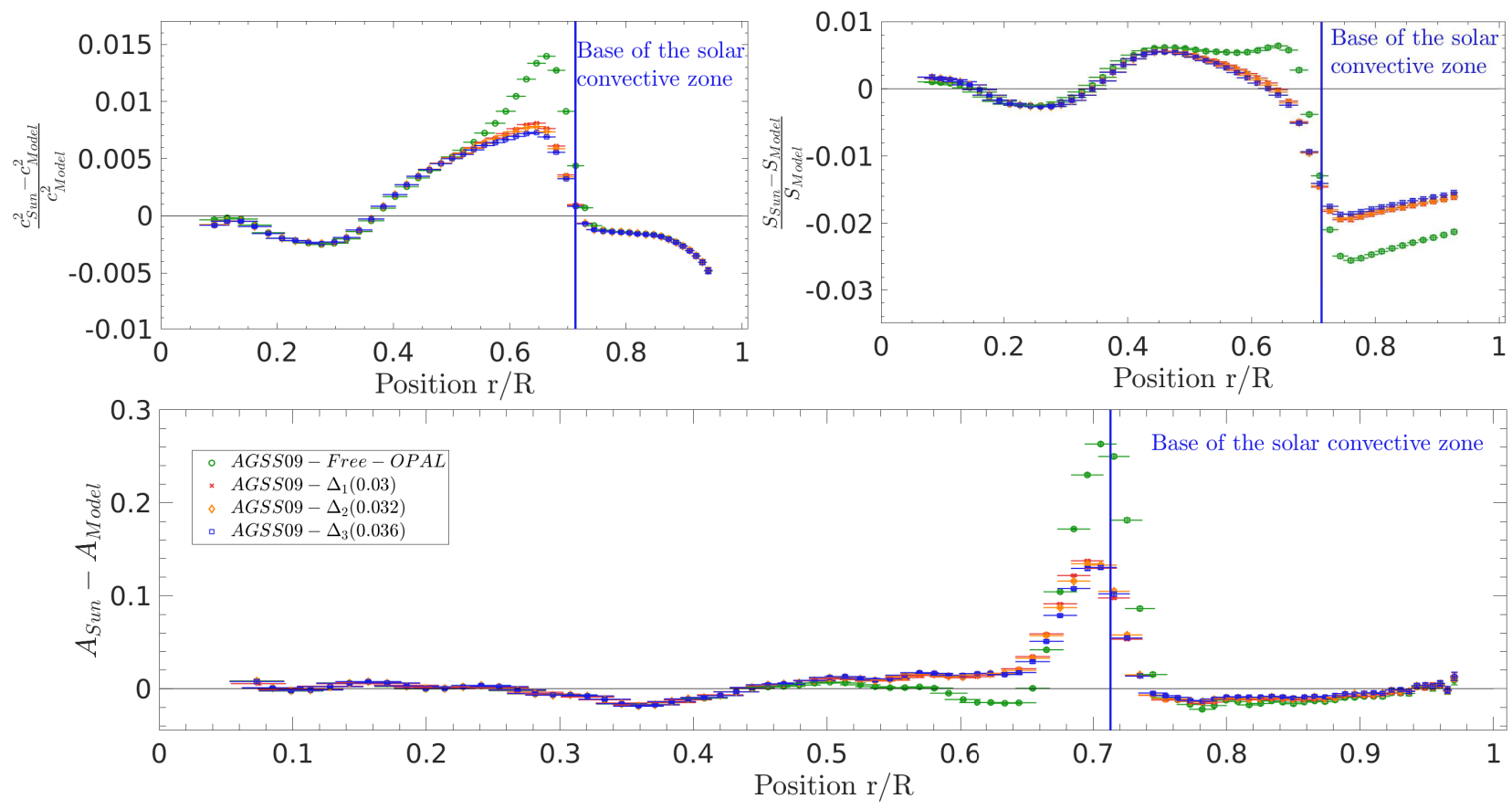

Fig. 6. Inversion results for models with modified opacities including a Gaussian peak at a fixed temperature with different widths but the same height (see text for details). Upper left: relative differences in squared adiabatic sound speed. Upper right: relative differences in entropy proxy, $S_{5 / 3}$. Lower panel: differences in Ledoux discriminant, $A$.

Table 2. Parameters of the solar models with modified opacity used in this study.

\begin{tabular}{rcccccccccc}
\hline \hline$(r / R)_{\mathrm{BCZ}}$ & $(m / M)_{\mathrm{CZ}}$ & $Y_{\mathrm{CZ}}$ & $Z_{\mathrm{CZ}}$ & $Y_{0}$ & $Z_{0}$ & EOS & Opacity & Abundances & Diffusion & Convection \\
\hline 0.7200 & 0.9776 & 0.2300 & 0.01372 & 0.2587 & 0.01519 & FreeEOS & OPLIB+log $T_{1}(6.25)$ & AGSS09 & Thoul & MLT \\
0.7165 & 0.9769 & 0.2302 & 0.01371 & 0.2586 & 0.01518 & FreeEOS & OPLIB+log $T_{2}(6.30)$ & AGSS09 & Thoul & MLT \\
0.7155 & 0.9766 & 0.2304 & 0.01371 & 0.2586 & 0.01515 & FreeEOS & OPLIB+log $T_{3}(6.35)$ & AGSS09 & Thoul & MLT \\
0.7195 & 0.9773 & 0.2303 & 0.01371 & 0.2588 & 0.01517 & FreeEOS & OPLIB+log $T_{4}(6.40)$ & AGSS09 & Thoul & MLT \\
0.7139 & 0.9768 & 0.2305 & 0.01368 & 0.2585 & 0.01514 & FreeEOS & OPLIB+ $\Delta_{1}$ & AGSS09 & Thoul & MLT \\
0.7136 & 0.9762 & 0.2301 & 0.01372 & 0.2585 & 0.01514 & FreeEOS & OPLIB+ $\Delta_{2}$ & AGSS09 & Thoul & MLT \\
0.7134 & 0.9761 & 0.2301 & 0.01363 & 0.2585 & 0.01514 & FreeEOS & OPLIB+ $\Delta_{3}$ & AGSS09 & Thoul & MLT \\
0.7174 & 0.9771 & 0.2301 & 0.01370 & 0.2585 & 0.01518 & FreeEOS & OPLIB+h1 & AGSS09 & Thoul & MLT \\
0.7165 & 0.9769 & 0.2302 & 0.01371 & 0.2586 & 0.01518 & FreeEOS & OPLIB+h $+h_{2}$ & AGSS09 & Thoul & MLT \\
0.7158 & 0.9761 & 0.2302 & 0.01371 & 0.2587 & 0.01518 & FreeEOS & OPLIB+h & AGSS09 & Thoul & MLT \\
0.7124 & 0.9758 & 0.2394 & 0.01355 & 0.2683 & 0.01496 & FreeEOS & OPAL+Poly & AGSS09 & Thoul & MLT \\
0.7104 & 0.9750 & 0.2332 & 0.01366 & 0.2608 & 0.01506 & FreeEOS & OPLIB+Poly & AGSS09 & Thoul & MLT \\
0.7089 & 0.9751 & 0.2354 & 0.01362 & 0.2634 & 0.01503 & FreeEOS & OPAS+Poly & AGSS09 & Thoul & MLT \\
0.7118 & 0.9755 & 0.2404 & 0.01353 & 0.2691 & 0.01493 & FreeEOS & OPAL+Poly2 & AGSS09 & Thoul & MLT \\
0.7056 & 0.9736 & 0.2495 & 0.01694 & 0.2791 & 0.01885 & FreeEOS & OPAL+Poly & GS98 & Thoul & MLT \\
\hline
\end{tabular}

temperature. We considered a height of $7 \%, 10 \%$, and $13 \%$ for the peak. The models are denoted respectively $h_{1}$ (red), $h_{2}$ (orange), and $h_{3}$ (blue) in Table 2. From Fig. 7 and Table 2, we see that the changes are minimal. Significant changes are only seen for the position of the BCZ, which is affected by the local steepening of the temperature gradient. This limited effect was already seen in Fig. 5 where it can be seen at $\log T=6.25$ and $\log T=6.3$ that the very localized opacity increase we considered had almost no effect. This is further confirmed in our tests when varying the height of the peak. However, this limited impact is only valid for such very localized changes and, as we see below, when more extended modifications are considered, the region at $\log T=6.3$ plays a crucial role.

In addition to localized increases, we tested a slightly more extended variation of the opacity, shown in Fig. 8. The explanation behind this modification is that most of the uncertainties on the opacities reside in the iron peak and around it, at $\log T=6.35$. However, due to the rapid increase in higher ionization states for most of the elements, the opacity computation are expected to be more robust as photon absorption becomes much less significant. Thus, we considered that the opacity difference would rapidly decrease with increasing temperature. We indicate models computed with this modification to the mean Rosseland opacity as "Poly" in the following figures, tables and discussions. The behaviour of this profile is not, however, by any means accurate and should only be seen as a qualitative estimate of what an opacity modification resulting from more accurate calculations could be. We did not apply any modification in the opacity profile at lower temperatures since they would be in the convective zone of the model, hence the sharp decrease in Fig. 8. However, this does not mean that such modifications are not expected ${ }^{4}$ and could not have an impact for stars other than the Sun.

4 They could potentially be larger than $10 \%$. 

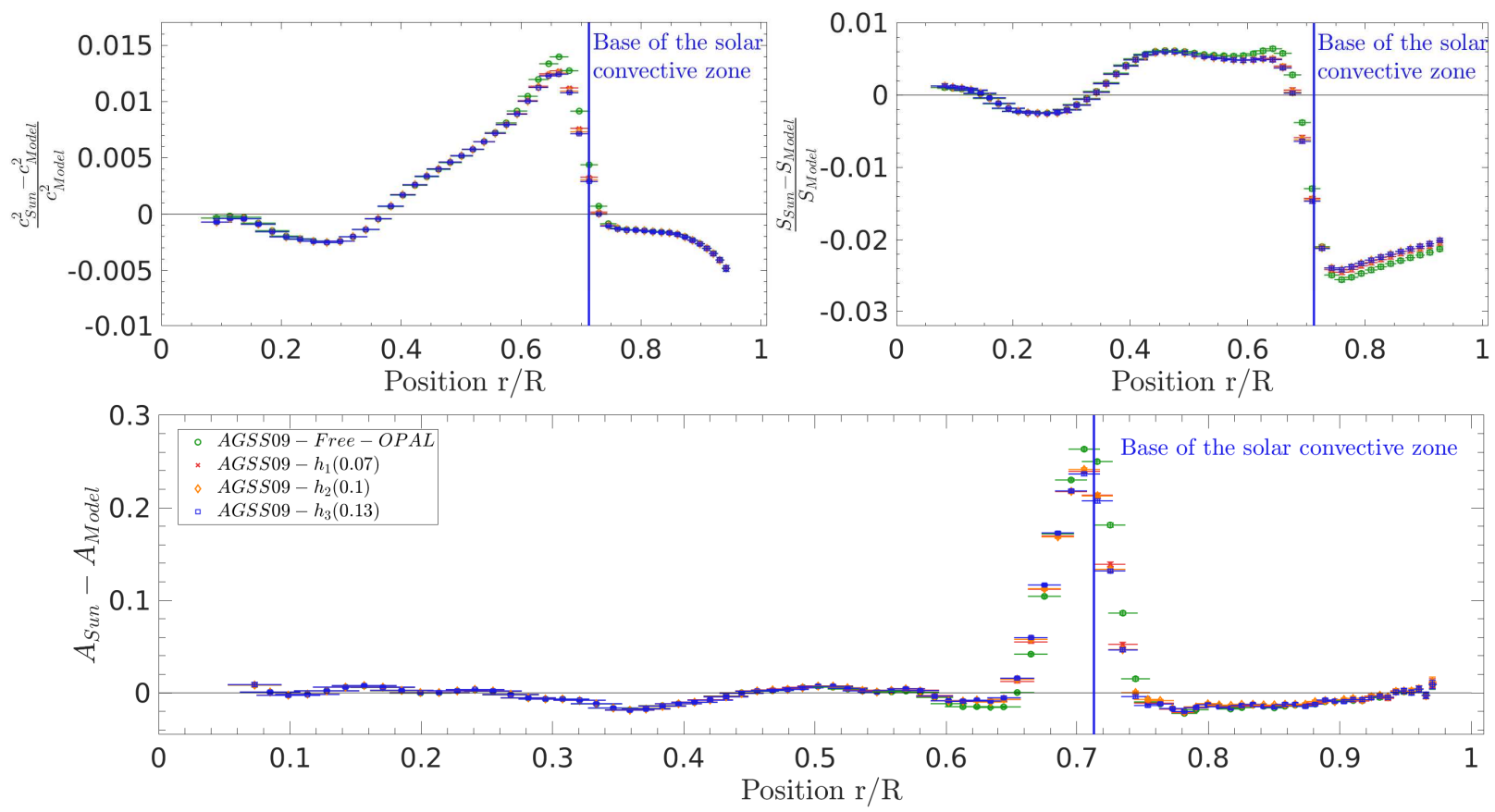

Fig. 7. Inversion results for models with modified opacities including a Gaussian peak at a fixed temperature with different heights and widths so as to keep the same increase at a fixed distance of the maximum (see text for details). Upper left: relative differences in squared adiabatic sound speed. Upper right: relative differences in entropy proxy, $S_{5 / 3}$. Lower panel: differences in Ledoux discriminant, $A$.

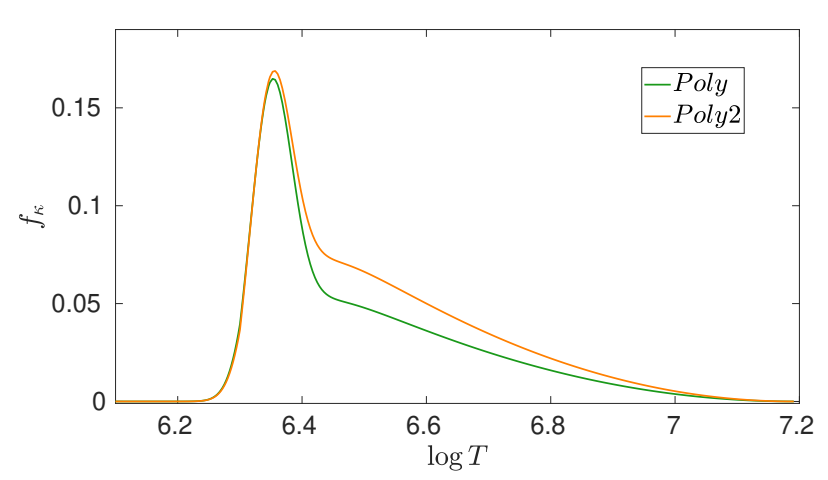

Fig. 8. Modification to the opacity profile used in the solar models denoted as "Poly" (green) and "Poly2" (orange) in Fig. 9 against $\log T$. $f_{K}$ is the increase in relative opacity applied during the evolution (see Eq. (2) and the enclosed discussion).

The changes brought by this opacity modification are quite impressive, showing for all models a drastic improvement in the height of the entropy plateau, in the sound speed, and in Ledoux discriminant profiles, as can be seen in Fig. 9. It is interesting to see that a small change around $3 \%$ between 0.4 and 0.5 solar radii has such a drastic effect on the sound speed profile. Moreover, this opacity modification has a non-negligible impact on the helium abundance in the convective zone, because a more widespread increase leads to a lower initial hydrogen abundance required to reproduce the solar luminosity at the solar age. We also note that our opacity modification has an amplitude similar to that of Christensen-Dalsgaard \& Houdek (2010), Ayukov \& Baturin (2011; see the dashed curve in Fig. 13 in the former and in Fig. 4 in the latter) at the BCZ, but with a much steeper decrease at higher temperature (see also Tripathy \& Christensen-Dalsgaard 1998, for a study in terms of opacity kernels.). Moreover, modifications of about 3\% are consistent with differences between various opacity tables (e.g.
Guzik et al. 2005, 2006), which can be used to estimate their optimistic uncertainties. At the level of accuracy of helioseismology, various hypotheses in the formalism of microscopic diffusion and variations in the equation of state can also induce differences between various modellers (see e.g. Montalban et al. 2006, and references therein for further discussions.). Typically, uncertainties on the diffusion velocities for iron and oxygen can reach values of about $35 \%$ resulting from assuming full ionization instead of partial ionization of the stellar material. Effects of radiative accelerations should remain small, but not fully negligible for iron (Turcotte et al. 1998; Gorshkov \& Baturin 2010), especially if the opacity of this element is underestimated.

Observing such changes from the introduction of a rather small additional component in the ad hoc increase in the opacity below the iron opacity peak around $\log T=6.35$ is surprising. In truth, it is due to changes in the opacity derivatives. The introduction of a localized increase unsurprisingly only impacts the models locally, while the addition of the polynomial decrease allows the Gaussian peak to impact the opacity profile at greater depth. Similarly, a constant increase in the opacity at all temperatures does not improve the properties of the models very much, except the helium abundance, because the temperature gradient is sensitive to the whole landscape of the opacities and thus to their derivatives (in agreement with Ayukov \& Baturin 2011, 2017; Colgan et al. 2016; Guzik et al. 2016). These variations of the solar calibrated models with respect to various opacity modifications clearly emphasize the advantage of taking into account multiple constraints and also illustrates the very constraining nature of the determination of the helium abundance in the solar convective zone.

All models built with the AGSS09 abundances show an agreement in sound speed with the Sun very similar to that of the ModelS from Christensen-Dalsgaard et al. (1996). Moreover, looking at Fig. 6 from Kosovichev (1999), the models built with the OPAL and OPAS opacities show a similar agreement in terms of Ledoux discriminant to ModelS. However, none of them 

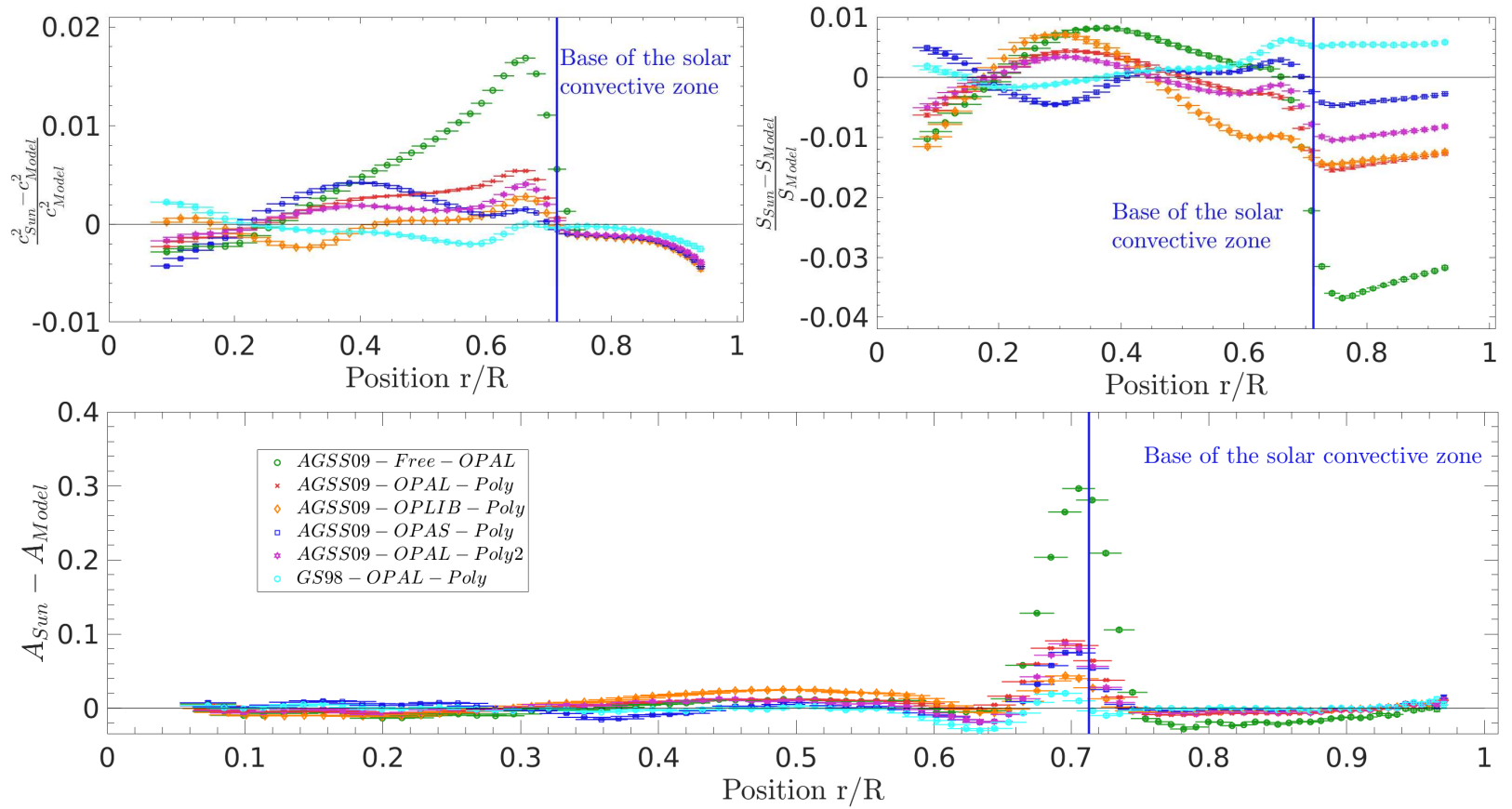

Fig. 9. Inversion results for models with modified opacities including a two-component modification for the opacity (see text for details). Upper left: relative differences in squared adiabatic sound speed. Upper right: relative differences in entropy proxy, $S_{5 / 3}$. Lower panel: differences in Ledoux discriminant, $A$.

has a satisfactory helium abundance and height of the entropy plateau. This illustrates the advantage of combining multiple inversions and show that the inversion of the sound speed alone can hide some compensation effects.

Such effects are illustrated by the model denoted Poly2, which considers a slightly slower decrease in the opacity modification with temperature, and the model built with the OPLIB opacities and the Poly modification in Fig. 9. As can be seen, both these models start inducing a slight disagreement around 0.65 solar radii in A. This effect is also present in ModelS, due to the higher metallicity but here it is a result of the opacity modification introduced in both models.

For the sake of comparison, we tested the extended opacity modification in a model using the GS98 abundances. We see that this model is in disagreement with helioseismic constraints. It shows a very deep BCZ, quite large discrepancies in Ledoux discriminant and sound speed around 0.6 solar radii, and a significant discrepancy in entropy proxy just below the convective zone (see Fig. 9). Moreover, it shows a quite low entropy proxy plateau, which is a clear sign of a too steep temperature gradient below the BCZ. However, the helium abundance in this model is in very good agreement with helioseismology and the discrepancies in the deeper radiative region, between 0.2 and 0.5 solar radii, in the inverted profiles are very small. This confirms that if an increased opacity is obtained in updated opacity tables, the higher metallicity solar abundances will disagree with helioseismology.

As for the models built with the AGSS09 abundances, we note that the OPLIB opacities induce a steeper temperature gradient near the BCZ despite their overall lower values in the solar radiative zone. Consequently, the model including the Poly modification built with the OPLIB opacities has a steeper temperature gradient near the envelope than the Poly model built with the OPAL opacities. Regarding the model Poly2, the steeper temperature gradient is a consequence of the higher amplitude of the changes made in the opacity profile near the BCZ. Both models closely resemble ModelS, with the exception of their lower helium abundance in the envelope. This very low abundance is particularly problematic for the OPLIB and OPAS opacities which, despite an increase in opacity over a broad domain, do not seem to be able to reproduce this constraint. This could indicate that they present a significantly larger underestimation of the opacity in the radiative region than other tables. Similarly, it should be noted that the model built with the OPAS opacity tables also shows larger discrepancies in the deep radiative region than the models built with the OPAL tables, and a too deep BCZ. From a theoretical point of view, we expect a certain amount of overshoot which would extend the region in which the temperature gradient is adiabatic. This in turn implies an even deeper limit of the convective zone, which would disagree even more with the helioseismology.

Combining all these analyses together confirms that no opacity tables seem to provide a satisfactory agreement with the Sun if the AGSS09 mixture is used. However, this also seems to indicate that changing the opacity only near the $\mathrm{BCZ}$ is insufficient to solve the solar modelling problem. Extending the opacity increase towards higher temperature can improve the agreement, but other constraints, such as the position of the BCZ and the helium abundance in the convective zone, have to be taken into account in the overall analysis. These constraints indicate that none of the models presented in this section is in satisfactory agreement with the Sun. This is especially problematic for the models computed with the OPAS and OPLIB tables, which are the latest generations of opacity tables. One way to partly solve this problem is to consider a constant increase in opacity of about 5\% over the whole solar structure, which is still close to the differences found between various former standard tables (Guzik et al. 2005, 2006). Such a modification does not have a great impact the profile inversions or the position of the $\mathrm{BCZ}$, but can strongly increase the helium abundance by about 0.006 , 
which would bring the OPAS models back in a range of helium values more acceptable (although still quite low).

\subsection{Impact of additional mixing and abundance changes}

In addition to uncertainties on opacities, standard solar models are also lacking a proper representation of the tachocline region, where additional mixing of the chemical elements is supposed to occur (Brun et al. 1999, 2002) and where the transition from the adiabatic to the radiative temperature gradient occurs in a smoother way than in models (Spiegel \& Zahn 1992; Monteiro et al. 1994a; Xiong \& Deng 2001; Rempel 2004; Hughes et al. 2007; Li \& Yang 2007; Yang \& Li 2007; Christensen-Dalsgaard et al. 2011; Zhang 2014; Hotta 2017).

In a previous study (Buldgen et al. 2017a), we showed that adding a localized additional diffusive mixing below the BCZ could reduce the discrepancies in the Ledoux discriminant inversions. Consequently, we decided to test models where extramixing would be used in addition to the extended opacity modification presented in Sect. 3.1. The behaviour and intensity of this mixing should be further investigated. Our results only depict the qualitative behaviour to be expected if such an extramixing is included. Comparisons with hydrodynamical simulations (e.g. Viallet et al. 2015) and models reproducing the solar rotation profile (Charbonnel \& Talon 2005; Eggenberger et al. 2005) could provide guidelines to empirical approaches for producing a smoother profile of the mean molecular weight, expected at the BCZ. On a longer timescale, improving the modelling of convection in stellar interiors altogether is the true concern of such studies. Currently, in addition to the opacity tables, the efficiency of chemical mixing in this region is the largest contributor to the uncertainties (Vinyoles et al. 2017).

For the tests presented here, we added the extra-mixing below the envelope as presented in Buldgen et al. (2017a), using a turbulent diffusion coefficient of the form

$D_{\text {Turb }}=D\left(\frac{\rho_{\mathrm{cz}}}{\rho(r)}\right)^{N}$,

with the free parameters $D$ and $N$ fixed respectively to 50 and 2 for the model AGSS09Ne-Poly-DTurb and 7500 and 3 for the model AGSS09Ne-Poly-DTurb-Prof. In Eq. (4), $\rho_{\mathrm{cz}}$ is the density value at the BCZ. The values of AGSS09Ne-Poly-DTurbProf are based on the models of Proffitt \& Michaud (1991) and Richard et al. (2005) that reproduced the lithium depletion in solar models (see also Richard et al. 1996; Piau \& Turck-Chièze 2001; Thévenin et al. 2017, for similar investigations using sound speed and lithium and beryllium depletion).

In addition to diffusive mixing, we considered models using instantaneous mixing below the BCZ. Model AGSS09Ne-PolyOv-Rad and AGSS09Ne-Poly-Ov-Ad included an extension of the mixed region over $0.3 \mathrm{H}_{\mathrm{p}}$, around the order of magnitude in Christensen-Dalsgaard et al. (2011). In model AGSS09NePoly-Ov-Ad, we used an adiabatic temperature gradient in the overshooting region, while for model AGSS09Ne-Poly-Ov-Rad, the temperature gradient was kept to its radiative value. The impact of these changes is discussed below. Other forms of mixing exist (see e.g. Zahn 1992). However, due to the uncertainties linked to the rotational transport in the Sun, such formalisms would need to be complemented by other mechanisms and further tested, which is beyond the scope of this study.

The extent of the mixed region is of 0.03-0.04 solar radii, similar to the estimated width of the tachocline (Corbard et al. 1999; Elliott \& Gough 1999; Hughes et al. 2007). We did not consider an additional overshoot in the models including diffusive mixing, as the opacity increase was sufficient to place the Schwarzschild limit quite low. This confirms that our opacity increase might be too large near the BCZ. We also included the $40 \%$ increase in neon abundance from Landi \& Testa (2015) and Young (2018), since it also provided a slight improvement.

The results of these inversions are presented in Fig. 10 for models using the Paquette et al. (1986; in orange) and Thoul et al. (1994; in red) diffusion coefficients, and one model including turbulent diffusion as in Buldgen et al. 2017a; in blue) whereas models including radiative or adiabatic overshooting with a fully mixed region (in orange and red, respectively) and the model including the coefficients of Proffitt \& Michaud (1991) for turbulent diffusion (in blue) are shown in Fig. 11. Additional information on each model can be found in Table 3 . We see from Figs. 10 and 11 that the agreement is further improved with the increase in neon abundance, both in the inversions and in the helium abundance in the envelope. It should be noted that the increase in helium is also partially due to the use of the SAHA-S equation of state and to the inclusion of turbulent diffusion. Using the Paquette et al. (1986) diffusion coefficients leads to a slightly higher surface helium abundance but at the expense of a slightly worse agreement of the inversions.

The models with turbulent diffusion show the best agreement with the Sun, especially (and unsurprisingly) the model using values of Proffitt \& Michaud (1991) for the parameters of Eq. (4). It provides a good value for the helium abundance and position of the BCZ while simultaneously showing quite good agreement with inversions. In comparison, the models including overshooting and instantaneous mixing perform poorly. However, the shift of the entropy plateau in the model including adiabatic overshooting illustrates the dependence of this quantity to the transition in temperature gradient just below the BCZ. Yet, while the entropy plateau is quite well reproduced, a glitch is generated deeper in the model, which is in clear contradiction with solar structure. This feature is not present in the model including radiative overshooting, demonstrating that this results from the temperature gradient. However, radiative overshooting appears to be unable to induce a shift in the entropy plateau, while it can reduce the errors in sound speed and Ledoux discriminant. The obvious and well-known conclusion of these observations is that the transition in temperature gradient is improperly reproduced by both models and should be somewhere in between these extremes. It is also worth mentioning that the models in Fig. 11 (except the standard model) reproduce the lithium destruction observed in the Sun, while the models in Fig. 10 do not.

Ultimately, our tests show the interplay between chemical mixing and opacities but also that the equation of state plays a non-negligible role at the accuracy level we aim for with solar models. Moreover, none of the models is able to fully reproduce each of the profiles and discrepancies are still present in the tachocline. This implies that the transition of the temperature gradient just below the envelope has a significant impact on these disagreements. Hence, our inversions should be coupled with phase shift analysis techniques as in Roxburgh \& Vorontsov (1994), Monteiro et al. (1994b) and Christensen-Dalsgaard et al. (2011) for an optimal diagnostic.

A less positive observation is made for the OPAS opacities (in purple in Fig. 10), which show a low helium abundance and a BCZ that is too deep, despite the inclusion of extra-mixing. The inversion results still show quite large discrepancies in the radiative region. Comparing Fig. 2 and the entropy inversions in Figs. 9 and 10, we see that the opacity increase has somewhat 
G. Buldgen et al.: Combining multiple structural inversions to constrain the solar modelling problem
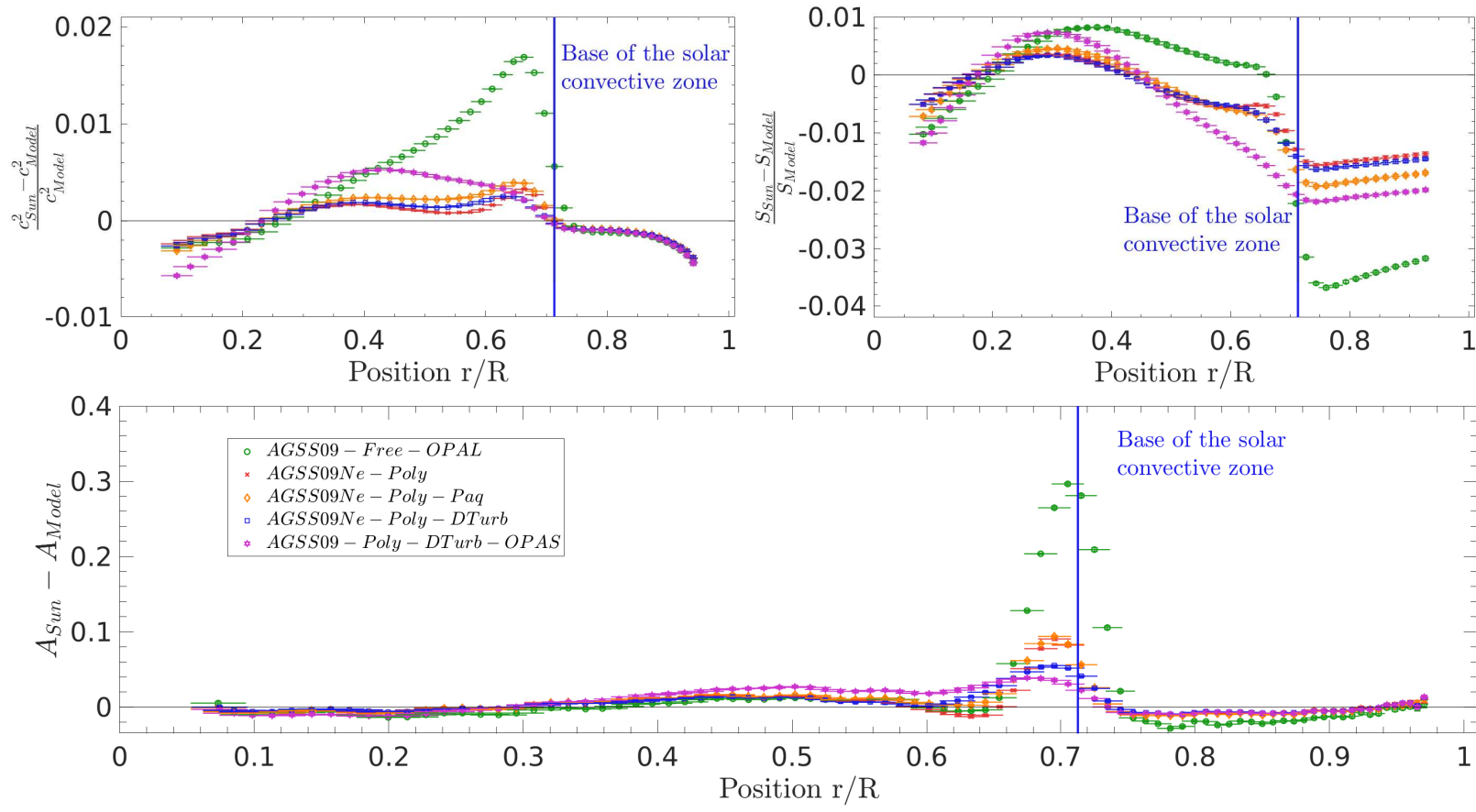

Fig. 10. Inversion results for models with modified opacities including a two component modification of opacity and various formalisms for the mixing of chemical elements (see text for details). Upper left: relative differences in squared adiabatic sound speed. Upper right: relative differences in entropy proxy, $S_{5 / 3}$. Lower panel: differences in Ledoux discriminant, $A$.
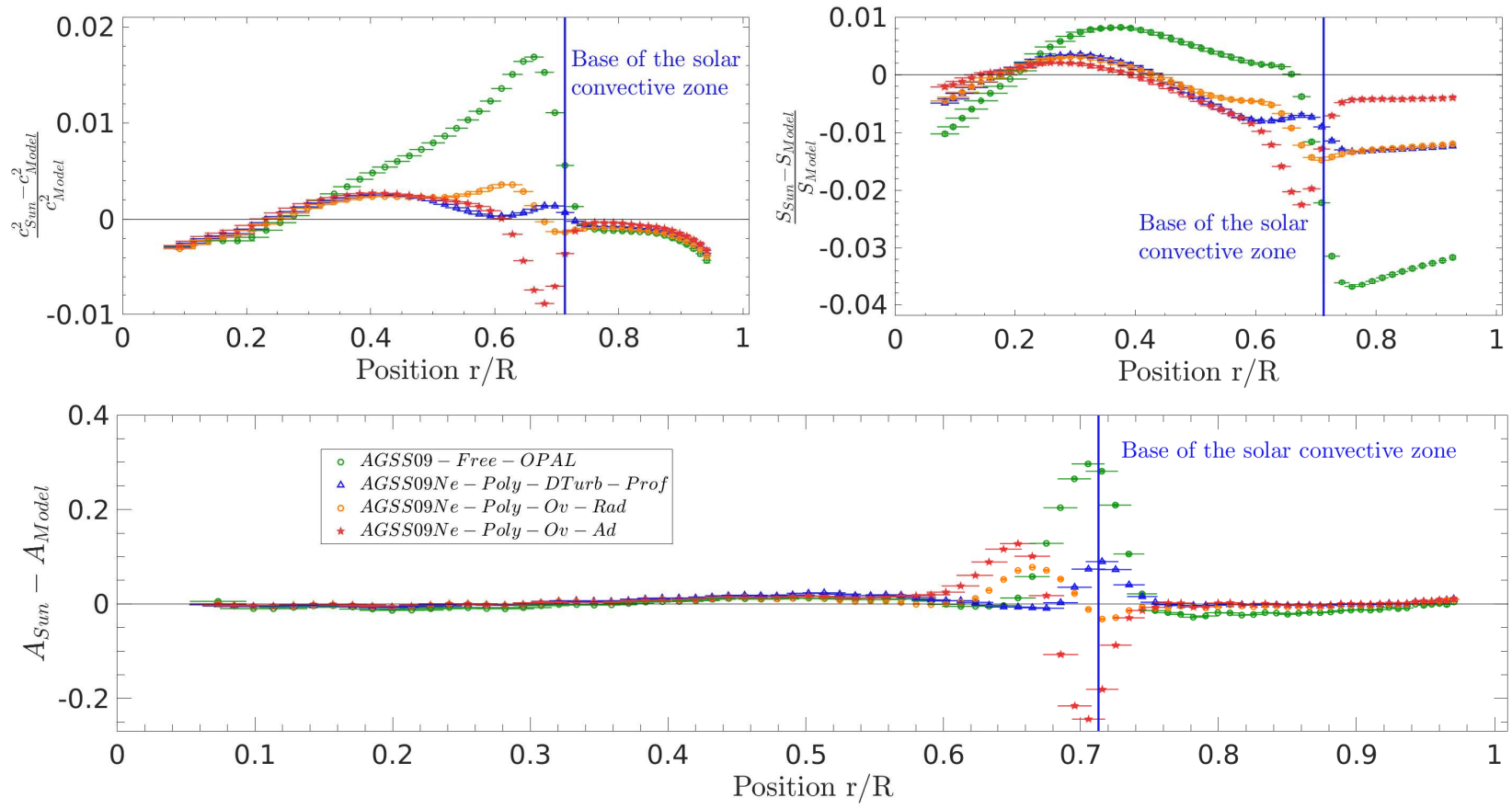

Fig. 11. Inversion results for models with modified opacities including a two component modification of opacity and including overshooting or turbulent diffusion following Proffitt \& Michaud (1991) for the mixing of chemical elements (see text for details). Upper left: relative differences in squared adiabatic sound speed. Upper right: relative differences in entropy proxy, $S_{5 / 3}$. Lower panel: differences in Ledoux discriminant, $A$.

improved the agreement in the radiative regions for the OPAL models, but has almost no effect on the OPAS models. Similar conclusions are drawn from sound speed inversions in Figs. 1, 9 and 10 .

Furthermore, the OPAS model is built without the neon increase because in their actual form, the OPAS opacities are limited to only one chemical composition. It is thus difficult to properly assess the impact of the changes in abundances and also to test how models built with the GS98 or GN93 composition would behave. Would they show problems in the Ledoux discriminant inversions similar to those found in models built with the OPLIB opacities? 
A\&A 621, A33 (2019)

Table 3. Parameters of the solar models with modified opacities and additional mixing used in this study.

\begin{tabular}{rcccccccccc}
\hline \hline$(r / R)_{\mathrm{BCZ}}$ & $(m / M)_{\mathrm{CZ}}$ & $Y_{\mathrm{CZ}}$ & $Z_{\mathrm{CZ}}$ & $Y_{0}$ & $Z_{0}$ & EOS & Opacity & Abundances & Diffusion & Convection \\
\hline 0.7122 & 0.9757 & 0.2416 & 0.01385 & 0.2692 & 0.01494 & SAHA-S & OPAL+Poly & AGSS09Ne & Thoul & MLT \\
0.7129 & 0.9761 & 0.2427 & 0.01383 & 0.2678 & 0.01483 & SAHA-S & OPAL+Poly & AGSS09Ne & Paquette & MLT \\
0.7106 & 0.9762 & 0.2425 & 0.01383 & 0.2685 & 0.01466 & SAHA-S & OPAL+Poly & AGSS09Ne & Thoul $+D_{\text {Turb }}$ & MLT \\
0.7106 & 0.9762 & 0.2374 & 0.01359 & 0.2645 & 0.01490 & SAHA-S & OPAS+Poly & AGSS09 & Thoul $+D_{\text {Turb }}$ & MLT \\
0.7121 & 0.9756 & 0.2460 & 0.01376 & 0.2696 & 0.01500 & SAHA-S & OPAL+Poly & AGSS09Ne & Thoul $+D_{\text {Turb }}-$ Prof & MLT \\
0.6871 & 0.9703 & 0.2437 & 0.01381 & 0.2692 & 0.01495 & SAHA-S & OPAL+Poly & AGSS09Ne & Thoul+Ov - Rad & MLT \\
0.6858 & 0.9697 & 0.2438 & 0.01381 & 0.2700 & 0.01506 & SAHA-S & OPAL+Poly & AGSS09Ne & Thoul+Ov - Ad & MLT \\
\hline
\end{tabular}

\section{Conclusion}

In this study, we have discussed the potential of combining multiple structural inversions to gain a deeper knowledge of the current inaccuracies of solar models and possible solutions to reconcile helioseismology with the AGSS09 abundances.

First, we presented in Sect. 2 the current state of the solar modelling problem using inversions of the sound speed, entropy proxy, and Ledoux discriminant for various combinations of standard physical ingredients of solar models. For each combination, we give the position of the $\mathrm{BCZ}$, the mass coordinate at this position and the initial and present-day photospheric abundances of helium and the heavy elements. We tested various opacity tables, formalisms for diffusion and convection, abundance tables, and equations of state. These calibrations show that no combination of current standard ingredients provides a satisfactory agreement for the AGSS09 models, but that higher metallicity models are not perfect either. We also find that using the latest opacity tables does not induce an unequivocal improvement. Although the $\mathrm{BCZ}$ and the inversion results are improved, it is made at the expense of the helium abundance. This is particularly problematic for the OPLIB opacities.

In Sect. 3, we investigated the potential of combining inversions to constrain changes in the opacity profile and chemical mixing at the BCZ to solve the solar modelling problem. We considered modifications to the opacity profile based on physical arguments. We started with localized modifications in the form of a Gaussian peak. We tested the positioning, width, and height of these increases and found the optimal positioning of the peak to be at $\log T=6.35$, in a temperature regime very close to that of an iron opacity peak. At this position, slight increases in the width of the peak had a limited effect on the results but a peak that is too broad would clearly impact them. Similarly, if the opacity increase is localized and located at $\log T=6.3$, the improvement in the models is quite scarce and variations in the height of the peak did not have a significant impact on the models. These results lend credence to the argument that part of the solar problem is linked to the iron opacity at this regime of physical conditions.

To further test opacity modifications, we implemented more elaborated variations, including potential smaller opacity underestimations at higher temperatures. While these tests remain purely speculative, they indicate that a relatively moderate opacity modification (of around $15 \%$ near the $\mathrm{BCZ}$ and $3 \%$ at higher temperature) can significantly increase the agreement between low-metallicity models and helioseismic results. Furthermore, analysing simultaneously the inversion results proves that a satisfactory agreement in one quantity can be disqualified by another, and thus help disentangle the multiple contributors to the observed discrepancies. This indicates that an approach using all the information of these inversions simultaneously could perhaps be used to derive a more precise estimation of the required opacity profile in the radiative zone, if an equation of state and a chemical composition profile for the model are assumed.

These tests on the opacity showed that the helium abundance in the convective zone remained quite low for low-metallicity models, even if an extended modification is used. Moreover, the $\mathrm{BCZ}$ quickly drops below the helioseismic value, implying an inadequate modification of the opacity. Reconciling these constraints with seismic estimates is only possible if a global opacity underestimation is considered instead of the profile we used or if other sources are invoked. Hence, we used models with a modified neon abundance according to recent estimates, a form of extra-mixing and used the equation of state that could push the results towards a better agreement in terms of helium and BCZ (the SAHA-S equation of state). For these cases, the improvement in the models is far more drastic; the helium abundance is higher due to the higher metallicity, the effects of the equation of state, and the extra-mixing. We considered both instantaneous mixing in the form of overshooting, using either the adiabatic or radiative temperature gradient, as well as turbulent diffusion using two sets of values for the parameters of the turbulent diffusion coefficient. It appears that the overall best agreement was obtained for a model including values from Proffitt \& Michaud (1991) for the parametric form of turbulent diffusion. However, the remaining discrepancies in the inversions seem to indicate that in addition to chemical mixing, a proper reproduction of the transition of the temperature gradients at the $\mathrm{BCZ}$ is also required. This confirms that more realistic implementation of overshooting may also influence the solar modelling problem. Moreover, the low position of the BCZ in these models may suggest that the modification of the opacity around the iron peak could be slightly too high if the updated neon abundances are used.

Overall, our tests suggest that the solar problem does not originate from one single source, but rather from various small contributors. First, additional investigations should be done to improve the formalism and hypotheses of microscopic diffusion (see e.g. Turcotte et al. 1998; Schlattl 2002; Gorshkov \& Baturin 2008, 2010). Comparisons between evolutionary codes from various groups (such as in Lebreton et al. 2007; Montalbán et al. 2007) could be pushed at the level of accuracy of helioseismology and provide crucial insights by pinpointing the intrinsic differences between each model, regardless of their (dis)agreement with the Sun. Second, further analyses using higher degree modes such as those derived from recent MDI datasets (Reiter et al. 2015) could prove very useful for revisiting studies such as those of Di Mauro et al. (2002), Lin \& Däppen (2005), Vorontsov et al. (2013) so as to constrain the upper parts of the convective envelope, the equation of state, and the 
abundance of heavy elements (see also Vorontsov et al. 2014; Buldgen et al. 2017b, for recent seismic determinations), which are key elements of the issue.

Moreover, a strong limitation of our study is the absence of a treatment of the transition in temperature gradient in the BCZ. Combining the inversions presented here to analyses as in Roxburgh \& Vorontsov (1994) and Christensen-Dalsgaard et al. (2011) could add strong constraints on the opacity changes just below the envelope. Coupling the two techniques could probably help disentangle the contributions of the opacity underestimation and the inadequate modelling of the temperature gradient transition in the tachocline to the overall discrepancies seen in the inverted profiles.

The recent investigations by Christensen-Dalsgaard et al. (2018) indicate that a density dependent diffusion coefficient coupled with significant modifications of the opacity profile (through rescaling the metal mixture and modifying the mean Rosseland opacity) can be used to eliminate the glitch of the tachocline in the solar data. From our tests, it appears that our formulation for the additional diffusive mixing coupled with our modification to the opacity profile does not erase completely the signal of the tachocline and is insufficient to reconcile the entropy plateau in the solar model with that of the Sun.

This could indicate that some entropy mixing could be present below the convective zone. However, this is purely speculative and further tests and comparisons should first be performed on the dependency of the form and intensity of the additional mixing introduced on the formalism and on the hypotheses and consistency of the mixing of chemical elements already present in the standard solar models.

In this sense, the study of Christensen-Dalsgaard et al. (2018) complements our work well. Here, we have been able to show that inputs from additional inversions can provide further constraints on the solar problem. However, we stress again that such studies should be complemented by in-depth investigations of the reliability of solar models as in Boothroyd \& Sackmann (2003) for various modelling groups.

With the recent $g$-modes detection by Fossat et al. (2017), further studies could try using these constraints along with the frequency ratios of Roxburgh \& Vorontsov (2003) and neutrino fluxes. However, such analyses should perhaps first remain unconnected, as the $g$-mode detection seems to be quite fragile (Schunker et al. 2018) and trying to combine all constraints might be very challenging.

Acknowledgements. A.M. and G.B. acknowledge support from the ERC Consolidator Grant funding scheme (project ASTEROCHRONOMETRY, G.A n. 772293). We gratefully acknowledge the support of the UK Science and Technology Facilities Council (STFC). S.J.A.J.S. is funded by the WalloniaBrussels Federation ARC grant for Concerted Research Actions. The work of V.K. Gryaznov, I.L. Iosilevskiy and A.N. Starostin was supported by RAS Scientific Program I.13 "Condensed matter and plasma under high energy density conditions". This article used an adapted version of InversionKit, a software developed within the HELAS and SPACEINN networks, funded by the European Commissions's Sixth and Seventh Framework Programmes. P.E. and G.M. acknowledge support from the Swiss National Science Foundation through the grant 200020-172505. The work of O.R. was supported by the "Programme National de Physique Stellaire" (PNPS) of CNRS/INSU co-funded by CEA and CNES. We wish to thank W. Däppen for fruitful discussions on questions related to the theory of equations of state used in stellar structure and evolution.

\section{References}

Adelberger, E. G., García, A., Robertson, R. G. H., et al. 2011, Rev. Mod. Phys., 83,195

Antia, H. M., \& Basu, S. 1994, A\&AS, 107, 421
Antia, H. M., \& Basu, S. 2005, ApJ, 620, L129

Asplund, M., Grevesse, N., Sauval, A. J., Allende Prieto, C., \& Kiselman, D. 2004, A\&A, 417, 751

Asplund, M., Grevesse, N., Sauval, A. J., Allende Prieto, C., \& Blomme, R. 2005, A\&A, 431, 693

Asplund, M., Grevesse, N., Sauval, A. J., \& Scott, P. 2009, ARA\&A, 47, 481

Ayukov, S. V., \& Baturin, V. A. 2011, J. Phys. Conf. Ser., 271, 012033

Ayukov, S. V., \& Baturin, V. A. 2017, Astron. Rep., 61, 901

Badnell, N. R., Bautista, M. A., Butler, K., et al. 2005, MNRAS, 360, 458

Bahcall, J. N., Huebner, W. F., Lubow, S. H., Parker, P. D., \& Ulrich, R. K. 1982, Rev. Mod. Phys., 54, 767

Bailey, J. E., Nagayama, T., Loisel, G. P., et al. 2015, Nature, 517, 3

Basu, S., \& Antia, H. M. 1995, MNRAS, 276, 1402

Basu, S., \& Antia, H. M. 1997, MNRAS, 287, 189

Basu, S., \& Antia, H. M. 2004, in SOHO 14 Helio- and Asteroseismology: Towards a Golden Future, ed. D. Danesy, ESA SP, 559, 317

Basu, S., \& Antia, H. M. 2008, Phys. Rep., 457, 217

Basu, S., Chaplin, W. J., Elsworth, Y., New, R., \& Serenelli, A. M. 2009, ApJ, 699, 1403

Baturin, V. A., Ayukov, S. V., Gryaznov, V. K., et al. 2013, in Progress in Physics of the Sun and Stars: A New Era in Helio- and Asteroseismology, eds. H. Shibahashi, \& A. E. Lynas-Gray, ASP Conf. Ser., 479, 11

Blancard, C., Cossé, P., \& Faussurier, G. 2012, ApJ, 745, 10

Blancard, C., Colgan, J., Cossé, P., et al. 2016, Phys. Rev. Lett., 117, 249501

Boothroyd, A. I., \& Sackmann, I.-J. 2003, ApJ, 583, 1004

Brown, T. M., \& Morrow, C. A. 1987, The Internal Solar Angular Velocity, eds.

B. R. Durney, \& S. Sofia, Astrophys. Space Sci. Library, 137, 7

Brun, A. S., Turck-Chièze, S., \& Zahn, J. P. 1999, ApJ, 525, 1032

Brun, A. S., Antia, H. M., Chitre, S. M., \& Zahn, J.-P. 2002, A\&A, 391, 725

Buldgen, G., Salmon, S. J. A. J., Godart, M., et al. 2017a, MNRAS, 472, L70

Buldgen, G., Salmon, S. J. A. J., Noels, A., et al. 2017b, MNRAS, 472, 751

Buldgen, G., Salmon, S. J. A. J., Noels, A., et al. 2017c, A\&A, 607, A58

Caffau, E., Ludwig, H.-G., Steffen, M., Freytag, B., \& Bonifacio, P. 2011, Sol. Phys., 268, 255

Canuto, V. M., \& Mazzitelli, I. 1991, ApJ, 370, 295

Canuto, V. M., \& Mazzitelli, I. 1992, ApJ, 389, 724

Canuto, V. M., Goldman, I., \& Mazzitelli, I. 1996, ApJ, 473, 550

Cassisi, S., Potekhin, A. Y., Pietrinferni, A., Catelan, M., \& Salaris, M. 2007, ApJ, 661, 1094

Charbonnel, C., \& Talon, S. 2005, Science, 309, 2189

Christensen-Dalsgaard, J., \& Däppen, W. 1992, A\&ARv, 4, 267

Christensen-Dalsgaard, J., \& Houdek, G. 2010, Ap\&SS, 328, 51

Christensen-Dalsgaard, J., Gough, D. O., \& Thompson, M. J. 1991, ApJ, 378, 413

Christensen-Dalsgaard, J., Däppen, W., Ajukov, S. V., et al. 1996, Science, 272, 1286

Christensen-Dalsgaard, J., Monteiro, M. J. P. F. G., Rempel, M., \& Thompson, M. J. 2011, MNRAS, 414, 1158

Christensen-Dalsgaard, J., Gough, D. O., \& Knudstrup, E. 2018, MNRAS, 477, 3845

Colgan, J., Kilcrease, D. P., Magee, N. H., et al. 2016, ApJ, 817, 116

Corbard, T., Blanc-Féraud, L., Berthomieu, G., \& Provost, J. 1999, A\&A, 344, 696

Cox, J. P., \& Giuli, R. T. 1968, Principles of Stellar Structure (New York: Gordon and Breach)

Däppen, W., Mihalas, D., Hummer, D. G., \& Mihalas, B. W. 1988, ApJ, 332, 261

Davies, G. R., Broomhall, A. M., Chaplin, W. J., Elsworth, Y., \& Hale, S. J. 2014, MNRAS, 439, 2025

Di Mauro, M. P., Christensen-Dalsgaard, J., Rabello-Soares, M. C., \& Basu, S. 2002, A\&A, 384, 666

Dziembowski, W. A., Pamyatnykh, A. A., \& Sienkiewicz, R. 1990, MNRAS, 244, 542

Dziembowski, W. A., Pamiatnykh, A. A., \& Sienkiewicz, R. 1991, MNRAS, 249, 602

Eggenberger, P., Maeder, A., \& Meynet, G. 2005, A\&A, 440, L9

Elliott, J. R., \& Gough, D. O. 1999, ApJ, 516, 475

Ferguson, J. W., Alexander, D. R., Allard, F., et al. 2005, ApJ, 623, 585

Fossat, E., Boumier, P., Corbard, T., et al. 2017, A\&A, 604, A40

Gabriel, M. 1996, A\&A, 309, 939

Gabriel, M. 1997, A\&A, 327, 771

García, R. A., Turck-Chièze, S., Jiménez-Reyes, S. J., et al. 2007, Science, 316, 1591

Gorshkov, A. B., \& Baturin, V. A. 2008, Astron. Rep., 52, 760

Gorshkov, A. B., \& Baturin, V. A. 2010, Ap\&SS, 328, 171

Grevesse, N., \& Noels, A. 1993, in Origin and Evolution of the Elements, eds.

N. Prantzos, E. Vangioni-Flam, \& M. Casse, 15

Grevesse, N., \& Sauval, A. J. 1998, Space Sci. Rev., 85, 161 
Grevesse, N., Scott, P., Asplund, M., \& Sauval, A. J. 2015, A\&A, 573, A27

Gryaznov, V. K., Ayukov, S. V., Baturin, V. A., et al. 2004, in Equation-ofState and Phase-Transition in Models of Ordinary Astrophysical Matter, eds. V. Celebonovic, D. Gough, \& W. Däppen, AIP Conf. Ser., 731, 147

Gryaznov, V. K., Ayukov, S. V., Baturin, V. A., et al. 2006, J. Phys. A: Math. Gen., 39, 4459

Gryaznov, V., Iosilevskiy, I., Fortov, V., et al. 2013, Contrib. Plasma Phys., 53, 392

Guzik, J. A., \& Mussack, K. 2010, ApJ, 713, 1108

Guzik, J. A., Watson, L. S., \& Cox, A. N. 2005, ApJ, 627, 1049

Guzik, J. A., Watson, L. S., \& Cox, A. N. 2006, Mem. Soc. Astron. It., 77, 389

Guzik, J. A., Fontes, C. J., Walczak, P., et al. 2016, IAU Focus Meeting, 29, 532

Hotta, H. 2017, ApJ, 843, 52

Houdek, G., Trampedach, R., Aarslev, M. J., \& Christensen-Dalsgaard, J. 2017, MNRAS, 464, L124

Hughes, D. W., Rosner, R., \& Weiss, N. O. 2007, The Solar Tachocline (Cambridge: Cambridge University Press)

Hummer, D. G., \& Mihalas, D. 1988, ApJ, 331, 794

Iglesias, C. A. 2015, MNRAS, 450, 2

Iglesias, C. A., \& Hansen, S. B. 2017, ApJ, 835, 284

Iglesias, C. A., \& Rogers, F. J. 1996, ApJ, 464, 943

Irwin, A.W. 2012, Astrophysics Source Code Library [record ascl:1211.002]

Kosovichev, A. G. 1988, Sov. Astron. Lett., 14, 145

Kosovichev, A. G. 1999, J. Comput. Appl. Math., 109, 1

Kosovichev, A. G., \& Fedorova, A. V. 1991, Sov. Astron., 35, 507

Landi, E., \& Testa, P. 2015, ApJ, 800, 110

Le Pennec, M., Turck-Chièze, S., Salmon, S., et al. 2015, ApJ, 813, L42

Lebreton, Y., Montalbán, J., Christensen-Dalsgaard, J., et al. 2007, in EAS Pub. Ser., eds. C. W. Straka, Y. Lebreton, \& M. J. P. F. G. Monteiro, 26, 155

Li, Y., \& Yang, J. Y. 2007, MNRAS, 375, 388

Lin, C.-H., \& Däppen, W. 2005, ApJ, 623, 556

Lynden-Bell, D., \& Ostriker, J. P. 1967, MNRAS, 136, 293

Mamajek, E. E., Prsa, A., Torres, G., et al. 2015, ArXiv e-prints [arXiv:1510.07674]

Marchenkov, K., Roxburgh, I., \& Vorontsov, S. 2000, MNRAS, 312, 39

Mihalas, D., Däppen, W., \& Hummer, D. G. 1988, ApJ, 331, 815

Mihalas, D., Hummer, D. G., Mihalas, B. W., \& Däppen, W. 1990, ApJ, 350, 300

Mondet, G., Blancard, C., Cossé, P., \& Faussurier, G. 2015, ApJs, 220, 2

Montalban, J., Miglio, A., Theado, S., Noels, A., \& Grevesse, N. 2006, Commun. Asteroseismolog., 147, 80

Montalbán, J., Théado, S., \& Lebreton, Y. 2007, in EAS Pub. Ser., eds. C. W. Straka, Y. Lebreton, \& M. J. P. F. G. Monteiro, 26, 167

Monteiro, M. J. P. F. G., Christensen-Dalsgaard, J., \& Thompson, M. J. 1994a, A\&A, 283, 247

Monteiro, M. J. P. F. G., Christensen-Dalsgaard, J., \& Thompson, M. J. 1994b, A\&A, 283, 247

Nahar, S. N., \& Pradhan, A. K. 2016, Phys. Rev. Lett., 116, 235003

Pain, J. C., Gilleron, F., \& Comet, M. 2017, ArXiv e-prints [arXiv:1706.01761]

Pain, J. C., Gilleron, F., \& Comet, M. 2018, ASP Conf. Ser., 515, 35

Paquette, C., Pelletier, C., Fontaine, G., \& Michaud, G. 1986, ApJS, 61, 177

Piau, L., \& Turck-Chièze, S. 2001, in From Darkness to Light: Origin and Evolution of Young Stellar Clusters, eds. T. Montmerle, \& P. André, ASP Conf. Ser., 243, 639
Pijpers, F. P., \& Thompson, M. J. 1994, A\&A, 281, 231

Potekhin, A. Y., Baiko, D. A., Haensel, P., \& Yakovlev, D. G. 1999, A\&A, 346, 345

Pradhan, A. K., \& Nahar, S. N. 2018, ASP Conf. Ser., 515, 79

Proffitt, C. R., \& Michaud, G. 1991, ApJ, 380, 238

Rabello-Soares, M. C., Basu, S., \& Christensen-Dalsgaard, J. 1999, MNRAS, 309, 35

Reese, D. R., Marques, J. P., Goupil, M. J., Thompson, M. J., \& Deheuvels, S. 2012, A\&A, 539, A63

Reiter, J., Rhodes, Jr., E. J., Kosovichev, A. G., et al. 2015, ApJ, 803, 92

Rempel, M. 2004, ApJ, 607, 1046

Richard, O., Vauclair, S., Charbonnel, C., \& Dziembowski, W. A. 1996, A\&A, 312, 1000

Richard, O., Dziembowski, W. A., Sienkiewicz, R., \& Goode, P. R. 1998, A\&A, 338, 756

Richard, O., Michaud, G., \& Richer, J. 2005, ApJ, 619, 538

Rogers, F. J., \& DeWitt, H. E. 1973, Phys. Rev. A, 8, 1061

Rogers, F. J., \& Nayfonov, A. 2002, ApJ, 576, 1064

Roxburgh, I. W., \& Vorontsov, S. V. 1994, MNRAS, 268, 880

Roxburgh, I. W., \& Vorontsov, S. V. 2003, A\&A, 411, 215

Schlattl, H. 2002, A\&A, 395, 85

Schlattl, H., Weiss, A., \& Ludwig, H.-G. 1997, A\&A, 322, 646

Schou, J., Antia, H. M., Basu, S., et al. 1998, ApJ, 505, 390

Schunker, H., Schou, J., Gaulme, P., \& Gizon, L. 2018, Sol. Phys., 293, 95

Scott, P., Asplund, M., Grevesse, N., Bergemann, M., \& Sauval, A. J. 2015a, A\&A, 573, A26

Scott, P., Grevesse, N., Asplund, M., et al. 2015b, A\&A, 573, A25

Scuflaire, R., Montalbán, J., Théado, S., et al. 2008a, Ap\&SS, 316, 149

Scuflaire, R., Théado, S., Montalbán, J., et al. 2008b, Ap\&SS, 316, 83

Serenelli, A. M., Basu, S., Ferguson, J. W., \& Asplund, M. 2009, ApJ, 705, L123

Sonoi, T., Belkacem, K., Dupret, M.-A., et al. 2017, A\&A, 600, A31

Spiegel, E. A., \& Zahn, J.-P. 1992, A\&A, 265, 106

Thévenin, F., Oreshina, A. V., Baturin, V. A., et al. 2017, A\&A, 598, A64

Thoul, A. A., Bahcall, J. N., \& Loeb, A. 1994, ApJ, 421, 828

Tripathy, S. C., \& Christensen-Dalsgaard, J. 1998, A\&A, 337, 579

Turcotte, S., Richer, J., Michaud, G., Iglesias, C. A., \& Rogers, F. J. 1998, ApJ, 504,539

Vernazza, J. E., Avrett, E. H., \& Loeser, R. 1981, ApJS, 45, 635

Viallet, M., Meakin, C., Prat, V., \& Arnett, D. 2015, A\&A, 580, A61

Vinyoles, N., Serenelli, A. M., Villante, F. L., et al. 2017, ApJ, 835, 202

Vorontsov, S. V., Baturin, V. A., \& Pamiatnykh, A. A. 1991, Nature, 349, 49

Vorontsov, S. V., Baturin, V. A., Ayukov, S. V., \& Gryaznov, V. K. 2013, MNRAS, 430, 1636

Vorontsov, S. V., Baturin, V. A., Ayukov, S. V., \& Gryaznov, V. K. 2014, MNRAS, 441, 3296

Xiong, D. R., \& Deng, L. 2001, MNRAS, 327, 1137

Yang, J. Y., \& Li, Y. 2007, MNRAS, 375, 403

Young, P. R. 2018, ApJ, 855, 15

Zaatri, A., Provost, J., Berthomieu, G., Morel, P., \& Corbard, T. 2007, A\&A, 469, 1145

Zahn, J.-P. 1992, A\&A, 265, 115

Zhang, Q. S. 2014, ApJ, 787, L28

Zhao, L., Eissner, W., Nahar, S. N., \& Pradhan, A. K. 2018, ASP Conf. Ser., 515, 89 